\title{
Synthesis of $( \pm)$-Bisavenanthramide B-6 by an Anionic Anhydride Mannich Reaction
}

Michael J. Di Maso, Gabriella M. Nepomuceno, Michael A. St. Peter, Haley H. Gitre, Kevin S. Martin, and Jared T. Shaw*

\section{Supporting Information Table of Contents}

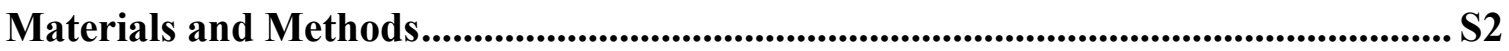

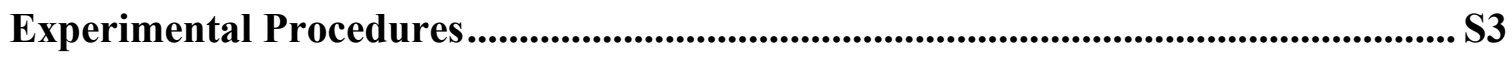

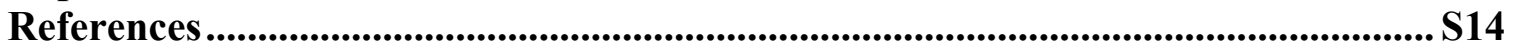

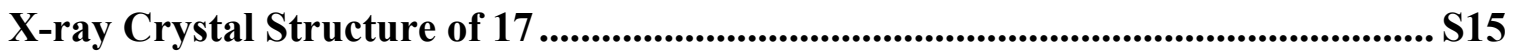

HPLC Traces................................................................................................................... S21

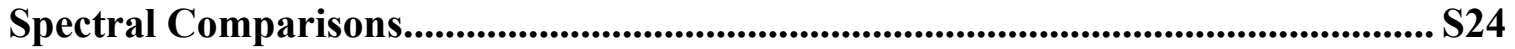

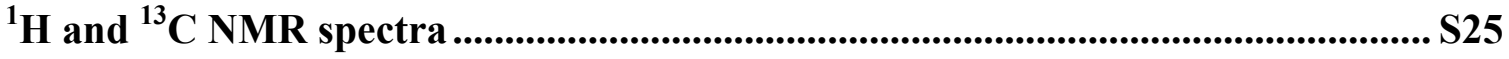




\section{Materials and Methods}

General considerations: All reagents and solvent were reagent grade and used without further purification unless otherwise specified. TLC was performed on silica-gel UV254

precoated glass backbone. The TLC spots were visualized using UV light, $\mathrm{KMnO}_{4}$ stain. Crude reaction products were purified using flash chromatography over silica gel (230 400 mesh, obtained from EM Science).

Instrumentation: All nuclear magnetic resonance (NMR) spectra were obtained using Varian UNITY INOVA NMR spectrometers (300 MHz, $400 \mathrm{MHz}$ or $600 \mathrm{MHz}$ ) or a Bruker $800 \mathrm{MHz}$ spectrometer. In the ${ }^{1} \mathrm{H} \mathrm{NMR}$ and ${ }^{13} \mathrm{C} \mathrm{NMR}$ spectra, $\mathrm{CDCl} 3(\delta 7.26$ ppm) was used as internal reference. Chemical shifts are reported in ppm and use the $\delta$ scale. The multiplicity, coupling constants (Hertz, $\mathrm{Hz}$ ) and number of protons are included in parentheses and are designated as s (singlet), d (doublet), t (triplet), $\mathrm{q}$ (quartet) $\mathrm{m}$ (multiplet), and dd (doublet of doublets). ${ }^{13} \mathrm{C}$ NMR spectra were acquired in the proton decoupled mode.

Accurate mass measurements were performed with a Thermo Fisher LTQOrbitrap spectrometer. Infrared (IR) spectra were recorded in cm-1 on a Bruker Tensor 27 FR-IR spectrophotometer equipped with a DTGS detector and Smart Orbit single bounce diamond ATR accessory. 


\section{Experimental Procedures}<smiles>COc1cc(/C=N/S(=O)(=O)CC[SiH3])ccc1OC(C)C</smiles>

(E)- $N$-(4-isopropoxy-3-methoxybenzylidene)-2-(trimethylsilyl)ethane-1-sulfonamide

(8). To a flame dried, 50-mL, round-bottomed flask were added 2-((2-

(trimethylsilyl)ethyl)sulfonyl)ethan-1-amine (4.23 g, $23.4 \mathrm{mmol}){ }^{1}$ 4-isopropoxy-3methoxybenzaldehyde $(5.03 \mathrm{~g}, 25.7 \mathrm{mmol}){ }^{2}$ and tetramethyl orthosilicate $(3.80 \mathrm{~mL}, 25.7$ mmol). The flask was fitted with a short path distillation head and heated at $110{ }^{\circ} \mathrm{C}$ for 4 hours. The reaction was diluted with EtOAc (approx. $20 \mathrm{~mL}$ ) and triturated into hexanes (approx. $250 \mathrm{~mL}$ ). The mixture was cooled to $-20{ }^{\circ} \mathrm{C}$ overnight and then filtered in a Buchner funnel to yield 8 (5.61 g, 67\%) as pale yellow crystals: mp 98.1-98.7 ${ }^{\circ} \mathrm{C} ;{ }^{1} \mathrm{H}$ NMR (600 MHz, $\left.\mathrm{CDCl}_{3}\right) \delta 8.89(\mathrm{~s}, 1 \mathrm{H}), 7.56(\mathrm{~d}, J=1.9 \mathrm{~Hz}, 1 \mathrm{H}), 7.44(\mathrm{dd}, J=8.3,1.9$ $\mathrm{Hz}, 1 \mathrm{H}), 6.96$ (d, $J=8.4 \mathrm{~Hz}, 1 \mathrm{H}), 4.71$ (hept, $J=6.1 \mathrm{~Hz}, 1 \mathrm{H}), 3.93$ (s, 3H), 3.16-3.10 (m, 2H), $1.44(\mathrm{~d}, J=6.1 \mathrm{~Hz}, 6 \mathrm{H}), 1.09-1.02(\mathrm{~m}, 2 \mathrm{H}), 0.06(\mathrm{~s}, 9 \mathrm{H}) ;{ }^{13} \mathrm{C} \mathrm{NMR}(150 \mathrm{MHz}$, $\left.\mathrm{CDCl}_{3}\right) \delta 171.6,154.2,150.6,129.1,125.0,113.0,110.9,71.7,56.3,49.1,22.0,9.7,-$ 1.8; IR (thin film) 2986, 2903, 1592, 1562, $1514 \mathrm{~cm}^{-1}$; AMM (ESI-TOF) $m / z$ : calcd for $\mathrm{C}_{16} \mathrm{H}_{28} \mathrm{NO}_{4} \mathrm{SSi}^{+}[\mathrm{M}+\mathrm{H}]^{+}$358.1503, found 358.1509.<smiles>COc1cc(/C=C(\CC(=O)O)C(=O)O)ccc1OC(C)C</smiles>

(E)-2-(4-isopropoxy-3-methoxybenzylidene)succinic acid (9a). To a flame dried, 250 $\mathrm{mL}$, round-bottomed flask 4-isopropoxy-3-methoxybenzaldehyde (5.01 g, $25.7 \mathrm{mmol})$, diethyl maleate $(4.6 \mathrm{~mL}, 28 \mathrm{mmol})$, tributylphosphane $(9.0 \mathrm{~mL}, 36 \mathrm{mmol})$, and $43 \mathrm{~mL}$ of toluene were added and heated to reflux for 24 hours. The reaction was tracked by mass spec. The toluene was removed in vaccuo and then $58 \mathrm{~mL}$ of THF, $28 \mathrm{~mL}$ of water, and 
lithium hydroxide $(3.72 \mathrm{~g}, 154 \mathrm{mmol})$ were added. The reaction was stirred for 16 hours. The reaction mixture was diluted with $100 \mathrm{~mL}$ of water and basified to $\mathrm{pH} 10$ with $18 \mathrm{M}$ $\mathrm{NaOH}$. The resulting solution was extracted with $\mathrm{CH}_{2} \mathrm{Cl}_{2}(3 \times 200 \mathrm{~mL})$. The aqueous layer was then acidified to $\mathrm{pH} 2$ with $12 \mathrm{M} \mathrm{HCl}$ and extracted with EtOAc ( 3 x $250 \mathrm{~mL})$. The EtOAc layers were combined, dried over $\mathrm{Na}_{2} \mathrm{SO}_{4}$, gravity filtered, and concentrated in vaccuo. The resulting material was triturated from hot ethyl acetate with hexanes. This yielded the di-acid 9a (6.70 g, 89\%) as an off white powder: mp 127.8-131.2 ${ }^{\circ} \mathrm{C} ;{ }^{1} \mathrm{H} \mathrm{NMR}$ $\left(600 \mathrm{MHz}, \mathrm{DMSO}-d_{6}\right) \delta 12.61(\mathrm{~s}, 2 \mathrm{H}), 7.64(\mathrm{~s}, 1 \mathrm{H}), 7.04(\mathrm{~d}, J=2.0 \mathrm{~Hz}, 1 \mathrm{H}), 7.02-6.93$ (m, 2H), 4.59 (hept, $J=6.0 \mathrm{~Hz}, 1 \mathrm{H}), 3.73$ (s, 3H), 3.40 (s, 2H), 1.24 (d, J=6.0 Hz, 6H); ${ }^{13} \mathrm{C}$ NMR (151 MHz, DMSO- $\left.d_{6}\right) \delta 172.8,169.2,149.8,148.0,140.6,127.8,125.3,122.8$, 114.9, 113.7, 70.5, 55.8, 34.0, 22.3; IR (thin film) 2925 (broad), 1704, 1666, $1510 \mathrm{~cm}^{-1}$; AMM (ESI-TOF) $m / z$ : calcd for $\mathrm{C}_{15} \mathrm{H}_{17} \mathrm{O}_{6}{ }^{-}[\mathrm{M}-\mathrm{H}]^{-} 293.1031$, found 293.1022.

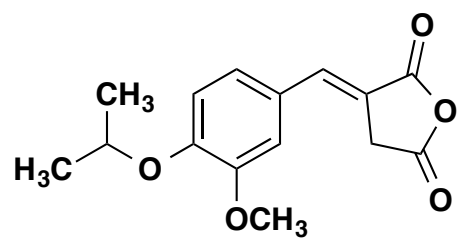

(E)-3-(4-isopropoxy-3-methoxybenzylidene)dihydrofuran-2,5-dione (9). To a flame dried, 250-mL, round-bottomed flask were added (E)-2-(4-isopropoxy-3methoxybenzylidene)succinic acid (5.61 g ,19.1 mmol), trifluoroacetic anhydride (13.1 $\mathrm{mL}, 95.1 \mathrm{mmol}$ ), and $80 \mathrm{~mL}$ of $\mathrm{CH}_{2} \mathrm{Cl}_{2}$. The reaction was stirred for 20 hours. The reaction was concentrated in vaccuo, then co-evaporated five times with toluene and four times with diethyl ether to yield anhydride 9 (5.03 g, 95\%) as an orange solid: mp 134.8$137.1{ }^{\circ} \mathrm{C} ;{ }^{1} \mathrm{H}$ NMR $\left(600 \mathrm{MHz}, \mathrm{CDCl}_{3}\right) \delta 7.71(\mathrm{~s}, 1 \mathrm{H}), 7.10(\mathrm{~d}, J=8.3 \mathrm{~Hz}, 1 \mathrm{H}), 6.99-6.95$ (m, 2H), 4.67 (hept, $J=6.2 \mathrm{~Hz}, 1 \mathrm{H}), 3.91$ (s, 3H), 3.81 (s, 2H), 1.43 (d, $J=6.2 \mathrm{~Hz}, 6 \mathrm{H})$; ${ }^{13} \mathrm{C}$ NMR $\left(151 \mathrm{MHz}, \mathrm{CDCl}_{3}\right) \delta$ 169.0, 166.8, 150.9, 150.4, 140.6, 125.9, 125.1, 116.1, 114.3, 114.0, 71.6, 56.3, 34.0, 22.1; IR (thin film) 2981, 2933, 1834, 1756, $1722 \mathrm{~cm}^{-1}$; AMM (ESI-TOF) $m / z$ : calcd for $\mathrm{C}_{15} \mathrm{H}_{20} \mathrm{NO}_{5}{ }^{+}\left[\mathrm{M}+\mathrm{NH}_{4}\right]^{+}$294.1336, found 294.1336. 


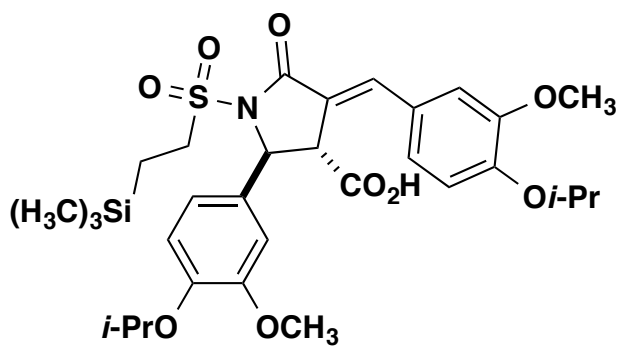

rel-(2S,3S)-4-((E)-4-isopropoxy-3-methoxybenzylidene)-2-(4-isopropoxy-3methoxyphenyl)-5-oxo-1-((2-(trimethylsilyl)ethyl)sulfonyl)pyrrolidine-3-carboxylic acid (11). To a flame dried, 500-mL, round-bottomed flask were added (E)-3-(4isopropoxy-3-methoxybenzylidene)dihydrofuran-2,5-dione (4.35 g, $15.7 \mathrm{mmol})$ and 80 $\mathrm{mL}$ of THF. The solution was cooled to $-78^{\circ} \mathrm{C}$. Sodium hydride $(903 \mathrm{mg}, 20.4 \mathrm{mmol}, 57$ $\mathrm{wt} \%$ ) was added and the solution was stirred for $5 \mathrm{~min}$. Then the reaction was diluted with THF (77 $\mathrm{mL})$ and $(E)-N$-(4-isopropoxy-3-methoxybenzylidene)-2-

(trimethylsilyl)ethane-1-sulfonamide $(5.58 \mathrm{~g}, 15.7 \mathrm{mmol})$ was added and the reaction was allowed to slowly warm to room temperature overnight. The reaction was monitored by NMR and was compete after 24 hours. The reaction was concentrated in vaccuo then taken up in $\mathrm{CH}_{2} \mathrm{Cl}_{2}(500 \mathrm{~mL})$ and washed with $3 \mathrm{M} \mathrm{HCl}(250 \mathrm{~mL})$. The aqueous layer was extracted with $\mathrm{CH}_{2} \mathrm{Cl}_{2}(2 \times 250 \mathrm{~mL})$. The organic layers were combined, dried over sodium sulfate, and concentrated in vaccuo. The resulting solid was purified by flash column chromatography $\left(95: 5 \mathrm{CH}_{2} \mathrm{Cl}_{2}: \mathrm{CH}_{3} \mathrm{OH}\right)$ to yield $11(7.01 \mathrm{~g}, 71 \%, 82: 18 \mathrm{dr})$ as a tan powder: ${ }^{1} \mathrm{H}$ NMR $\left(600 \mathrm{MHz}, \mathrm{CDCl}_{3}\right) \delta 7.71(\mathrm{~d}, J=1.6 \mathrm{~Hz}, 1 \mathrm{H}), 7.17-7.11(\mathrm{~m}, 2 \mathrm{H})$, $6.89(\mathrm{~d}, J=8.4 \mathrm{~Hz}, 1 \mathrm{H}), 6.83-6.79(\mathrm{~m}, 3 \mathrm{H}), 5.49 \mathrm{j}(\mathrm{s}, 1 \mathrm{H}), 4.60$ (hept, $J=5.9 \mathrm{~Hz}, 1 \mathrm{H})$, 4.47 (hept, $J=6.1 \mathrm{~Hz}, 1 \mathrm{H}), 4.09(\mathrm{~s}, 1 \mathrm{H}), 3.83(\mathrm{~s}, 3 \mathrm{H}), 3.78$ (s, 3H), 3.44 (ddd, $J=14.0$, 14.0, 4.3 Hz, 1H), 3.04 (ddd, $J=13.9,13.8,4.3 \mathrm{~Hz}, 1 \mathrm{H}), 1.37$ (d, $J=6.1, \mathrm{~Hz}, 6 \mathrm{H}), 1.33$ $(\mathrm{d}, J=6.1 \mathrm{~Hz}, 6 \mathrm{H}), 0.94-0.87(\mathrm{~m}, 2 \mathrm{H}),-0.05(\mathrm{~s}, 9 \mathrm{H}) ;{ }^{*}{ }^{13} \mathrm{C} \mathrm{NMR}\left(151 \mathrm{MHz}, \mathrm{CDCl}_{3}\right) \delta$ 174.1, 167.5, 150.4, 150.03, 149.98, 147.8, 139.6, 132.4, 125.8, 125.3, 121.9, 118.1, $115.1,114.1,113.4,110.0,71.3,71.2,62.8,56.1,56.0,51.6,50.5,22.0$ (2), 21.93, 21.89, 9.0, -2.1; IR (thin film) 3242, 2977, 1720, 1640, $1598 \mathrm{~cm}^{-1}$; AMM (ESI-TOF) $\mathrm{m} / \mathrm{z}$ : calcd for $\mathrm{C}_{31} \mathrm{H}_{44} \mathrm{NO}_{9} \mathrm{SSi}^{+}[\mathrm{M}+\mathrm{H}]^{+}$634.2501, found 634.2516.

*The carboxylic acid proton signal is not observed in $\mathrm{CDCl}_{3}$.

† The isopropyl methyl carbons are observed to be diastereotopic. 


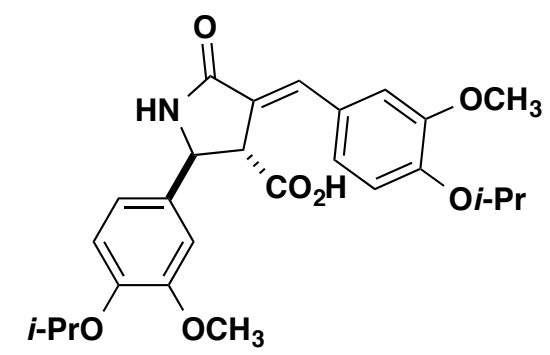

rel-(2S,3S)-4-((E)-4-isopropoxy-3-methoxybenzylidene)-2-(4-isopropoxy-3-

methoxyphenyl)-5-oxopyrrolidine-3-carboxylic acid (12). To a flame dried, 25-mL, round-bottomed flask were added $11(0.736 \mathrm{~g}, 1.16 \mathrm{mmol})$ and $11.7 \mathrm{~mL}$ of $1.0 \mathrm{M}$ TBAF in $\mathrm{CH}_{2} \mathrm{Cl}_{2}$. The reaction was stirred for 18 hours and then concentrated in vacuo. The resulting residue was portioned between $\mathrm{Et}_{2} \mathrm{O}(200 \mathrm{~mL})$ and water $(30 \mathrm{~mL})$. The layers were separated and the organic layer was washed with water $(7 \times 30 \mathrm{~mL})$ to remove excess TBAF. The organic layer was dried over $\mathrm{Na}_{2} \mathrm{SO}_{4}$, gravity filtered, and concentrated in vacuo. The solid was recrystallized from $\mathrm{Et}_{2} \mathrm{O}$ to yield 12 (502 $\left.\mathrm{mg}, 92 \%\right)$ as a white solid: mp 192.4-193.5 ${ }^{\circ} \mathrm{C} ;{ }^{1} \mathrm{H}$ NMR (600 MHz, $\left.\mathrm{CDCl}_{3}\right) \delta 8.01$ (s, 1H), 7.56 (s, 1H), 7.08-7.01 (m, 2H), $6.86(\mathrm{~d}, J=8.3 \mathrm{~Hz}, 1 \mathrm{H}), 6.80(\mathrm{~d}, J=8.1 \mathrm{~Hz}, 1 \mathrm{H}), 6.77-6.72(\mathrm{~m}$, 2H), 4.98 (s, 1H), 4.57 (hept, $J=6.2 \mathrm{~Hz}, 1 \mathrm{H}), 4.47$ (hept, $J=6.1 \mathrm{~Hz}, 1 \mathrm{H}), 4.02$ (s, 1H), $3.83(\mathrm{~s}, 3 \mathrm{H}), 3.78(\mathrm{~s}, 3 \mathrm{H}), 1.36(\mathrm{~d}, J=6.0 \mathrm{~Hz}, 6 \mathrm{H}), 1.33(\mathrm{~d}, J=6.1 \mathrm{~Hz}, 6 \mathrm{H})$; ${ }^{13} \mathrm{C} \mathrm{NMR}$ $\left(151 \mathrm{MHz}, \mathrm{CDCl}_{3}\right) \delta 175.1,173.4,150.8,150.1,149.2,147.5,136.4,134.2,126.7,124.6$, $123.8,117.6,115.8,114.4,113.0,109.4,71.6,71.3,60.0,56.1,56.1,53.4,22.2$, 22.1; IR (thin film) 3270, 2974, 1696, 1624, $1510 \mathrm{~cm}^{-1}$; AMM (ESI-TOF) $\mathrm{m} / \mathrm{z}$ : calcd for $\mathrm{C}_{26} \mathrm{H}_{32} \mathrm{NO}_{7}^{+}[\mathrm{M}+\mathrm{H}]^{+}$470.2173, found 470.2177.

*The carboxylic acid proton signal is not observed in $\mathrm{CDCl}_{3}$. 


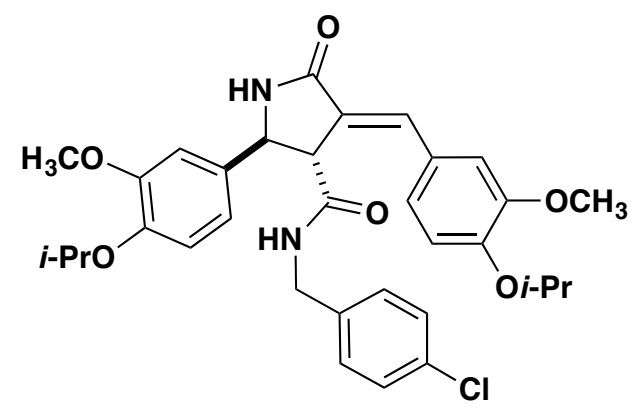

rel-(2S,3S)- $N$-(4-chlorobenzyl)-4-((E)-4-isopropoxy-3-methoxybenzylidene)-2-(4isopropoxy-3-methoxyphenyl)-5-oxopyrrolidine-3-carboxamide (17). To a flame dried, 7-mL vial were added $12(50 \mathrm{mg}, 0.11 \mathrm{mmol})$ and $0.60 \mathrm{~mL}$ of THF. The solution was cooled to $-10{ }^{\circ} \mathrm{C}$ with an ice-ethanol bath. After cooling, $N$-methylmorpholine (24 $\mu \mathrm{L}, 0.22 \mathrm{mmol})$ and isobutyl chloroformate $(16 \mu \mathrm{L}, 0.12 \mathrm{mmol})$ were added sequentially. The solution was stirred for 10 minutes and then 4-chlorobenzylamine (16 $\mu \mathrm{L}, 0.13 \mathrm{mmol})$. The reaction was allowed to slowly warm to room temperature overnight. The reaction was concentrated in vacuo and dissolved in EtOAc $(5 \mathrm{~mL})$ and water $(5 \mathrm{~mL})$. The layers were separated and the aqueous layer was extracted with EtOAc $(2 \times 5 \mathrm{~mL})$. The combined organic layers were dried over $\mathrm{Na}_{2} \mathrm{SO}_{4}$, gravity filtered, and concentrated in vacuo to yield $17(37 \mathrm{mg}, 57 \%)$ as a thin film. A sample of this compound was dissolved in $\mathrm{CH}_{3} \mathrm{OH}$. The solvent was slowly evaporated to provide crystals suitable for x-ray diffraction: $\mathrm{mp}$ 155.3-157.4 ${ }^{\circ} \mathrm{C} ;{ }^{1} \mathrm{H} \mathrm{NMR}\left(600 \mathrm{MHz}, \mathrm{CDCl}_{3}\right) \delta$ $7.19(\mathrm{~d}, J=8.2 \mathrm{~Hz}, 1 \mathrm{H}), 6.98-6.91(\mathrm{~m}, 3 \mathrm{H}), 6.89(\mathrm{~s}, 2 \mathrm{H}), 6.80(\mathrm{~d}, J=8.0 \mathrm{~Hz}, 2 \mathrm{H}), 6.65-$ $6.55(\mathrm{~m}, 2 \mathrm{H}), 6.12(\mathrm{~s}, 1 \mathrm{H}), 4.94(\mathrm{~s}, 1 \mathrm{H}), 4.54-4.47(\mathrm{~m}, 2 \mathrm{H}), 4.29(\mathrm{~d}, J=6.2 \mathrm{~Hz}, 1 \mathrm{H})$, 3.99-3.95 (m, 1H), $3.88(\mathrm{~s}, 3 \mathrm{H}), 3.83(\mathrm{~d}, J=6.7 \mathrm{~Hz}, 1 \mathrm{H}), 3.60(\mathrm{~s}, 3 \mathrm{H}), 1.39$ (d, $J=6.1$ $\mathrm{Hz}, 3 \mathrm{H}), 1.35(\mathrm{~d}, J=6.1 \mathrm{~Hz}, 3 \mathrm{H}), 1.29$ (d, $J=6.1 \mathrm{~Hz}, 6 \mathrm{H}) ;{ }^{13} \mathrm{C} \mathrm{NMR}\left(151 \mathrm{MHz}, \mathrm{CDCl}_{3}\right)$ $\delta 173.2,171.3,151.1,150.0,149.4,147.6,136.4,135.0,132.9,129.1,128.8,128.4$, $125.7,125.2,123.7,117.3,116.0,113.8,112.5,109.7,71.6,71.2,56.3,56.0,44.4,42.5$, 28.1, 22.09, 22.07, 22.05, 22.0; ${ }^{\star}$ IR (thin film) 3291, 3187, 2978, 2936, 1696, 1666, 1637 $\mathrm{cm}^{-1}$; AMM (ESI-TOF) $\mathrm{m} / z$ : calcd for $\mathrm{C}_{33} \mathrm{H}_{38} \mathrm{ClN}_{2} \mathrm{O}_{6}{ }^{+}[\mathrm{M}+\mathrm{H}]^{+}$593.2413, found 593.2449 .

*The Two amide $(\mathrm{N}-\mathrm{H})$ protons are not observed in $\mathrm{CDCl}_{3}$. † The isopropyl methyl carbons are observed to be diastereotopic. 


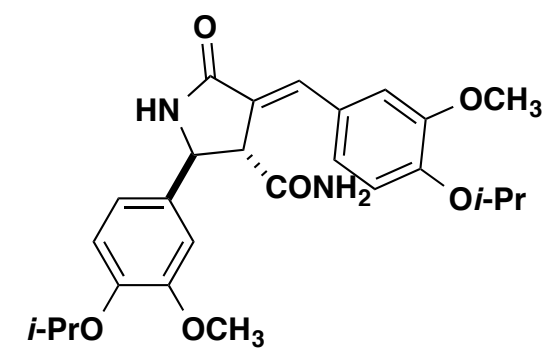

rel-(2S,3S)-4-((E)-4-isopropoxy-3-methoxybenzylidene)-2-(4-isopropoxy-3-

methoxyphenyl)-5-oxopyrrolidine-3-carboxamide (7). To a flame dried, 25-mL, roundbottomed flask were added $12(1.0 \mathrm{~g}, 2.1 \mathrm{mmol})$ and $10.7 \mathrm{~mL}$ of THF. The solution was cooled to $-10{ }^{\circ} \mathrm{C}$ with an ice-ethanol bath. After cooling, $N$-methylmorpholine $(0.47 \mathrm{~mL}$, $4.3 \mathrm{mmol})$ and isobutyl chloroformate $(0.30 \mathrm{~mL}, 2.3 \mathrm{mmol})$ were added sequentially by syringe in single portions. The solution was stirred for 10 minutes and then aqueous ammonium hydroxide $(0.43 \mathrm{~mL}, 6.4 \mathrm{mmol}, 14.8 \mathrm{M})$ was added. The reaction was allowed to warm to room temperature and stirred for 4 hours. The reaction was concentrated in vacuo and diluted with EtOAc $(50 \mathrm{~mL})$ and water $(50 \mathrm{~mL})$. The layers were separated and the aqueous layer was extracted with EtOAc $(2 \times 50 \mathrm{~mL})$. The combined organic layers were dried over $\mathrm{Na}_{2} \mathrm{SO}_{4}$, gravity filtered, and concentrated in vacuo to provide 7 (0.73 g, 73\%) as a yellow solid: mp 148.3-152.5 ${ }^{\circ} \mathrm{C}$; ${ }^{1} \mathrm{H}$ NMR (600 $\left.\mathrm{MHz}_{\mathrm{CDCl}}\right) \delta 7.52(\mathrm{~s}, 1 \mathrm{H}), 7.36(\mathrm{~s}, 1 \mathrm{H}), 6.99(\mathrm{~d}, J=8.5 \mathrm{~Hz}, 1 \mathrm{H}), 6.96(\mathrm{~s}, 1 \mathrm{H}), 6.86-$ $6.80(\mathrm{~m}, 4 \mathrm{H}), 6.32(\mathrm{~s}, 1 \mathrm{H}), 5.85(\mathrm{~s}, 1 \mathrm{H}), 4.94(\mathrm{~s}, 1 \mathrm{H}), 4.56$ (hept, $J=6.1 \mathrm{~Hz}, 1 \mathrm{H}), 4.48$ (hept, $J=6.1 \mathrm{~Hz}, 1 \mathrm{H}), 3.93(\mathrm{~s}, 1 \mathrm{H}), 3.82(\mathrm{~s}, 3 \mathrm{H}), 3.80(\mathrm{~s}, 3 \mathrm{H}), 1.37(\mathrm{~d}, J=6.1 \mathrm{~Hz}, 6 \mathrm{H})$, $1.34(\mathrm{~d}, J=6.2 \mathrm{~Hz}, 6 \mathrm{H}) ;{ }^{13} \mathrm{C} \mathrm{NMR}\left(151 \mathrm{MHz}, \mathrm{CDCl}_{3}\right) \delta 173.1,171.6,150.9,150.2$, 149.4, 147.4, 136.5, 135.0, 126.4, 124.4, 124.4, 117.6, 115.9, 114.4, 113.2, 109.4, 71.6, 71.3, 59.9, 56.2, 56.1, 55.6, 22.2, 22.1; IR (thin film) 3287, 2175, 2974, 1689, 1644, 1600 $\mathrm{cm}^{-1}$; AMM (ESI-TOF) $\mathrm{m} / z$ : calcd for $\mathrm{C}_{26} \mathrm{H}_{33} \mathrm{~N}_{2} \mathrm{O}_{6}{ }^{+}[\mathrm{M}+\mathrm{H}]^{+} 469.2333$, found 469.2333. 


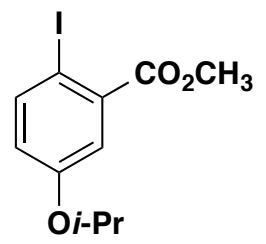

methyl 2-iodo-5-isopropoxybenzoate (6). To a flame-dried, 100-mL, round-bottomed flask were added methyl 5-hydroxy-2-iodobenzoate (3.86 g, $13.9 \mathrm{mmol}),{ }^{3} \mathrm{~K}_{2} \mathrm{CO}_{3}(2.89 \mathrm{~g}$, $20.9 \mathrm{mmol})$ and $14 \mathrm{~mL}$ of DMF. 2-Bromopropane $(1.96 \mathrm{~mL}, 20.9 \mathrm{mmol})$ was added via syringe and the reaction was heated at $80{ }^{\circ} \mathrm{C}$ overnight. The reaction was allowed to cool to room temperature and diluted with water $(100 \mathrm{~mL})$. The solution was extracted with EtOAc $(3 \times 100 \mathrm{~mL})$. The combined organic layers were washed with water $(3 \times 20 \mathrm{~mL})$, brine ( $20 \mathrm{~mL}$ ), dried over $\mathrm{Na}_{2} \mathrm{SO}_{4}$, gravity filtered, and concentrated in vacuo to provide $6(3.64 \mathrm{~g}, 82 \%)$ as a colorless oil: ${ }^{1} \mathrm{H}$ NMR $\left(600 \mathrm{MHz}, \mathrm{CDCl}_{3}\right) \delta 7.83(\mathrm{~d}, J=8.7 \mathrm{~Hz}, 1 \mathrm{H})$, 7.35 (d, $J=3.3 \mathrm{~Hz}, 1 \mathrm{H}), 6.74$ (dd, $J=8.7,3.0 \mathrm{~Hz}, 1 \mathrm{H}$ ), 4.56 (hept, $J=6.1 \mathrm{~Hz}, 1 \mathrm{H}$ ), 3.94 (s, 3H), $1.34(\mathrm{~d}, J=6.0 \mathrm{~Hz}, 6 \mathrm{H}) ;{ }^{13} \mathrm{C} \mathrm{NMR}\left(151 \mathrm{MHz}, \mathrm{CDCl}_{3}\right) \delta$ 166.8, 157.8, 141.9, 135.8, 120.9, 118.4, 82.0, 70.4, 52.5, 21.8; IR (thin film) 2982, 2956, 1734, 1592, 1567 $\mathrm{cm}^{-1}$; AMM (ESI-TOF) $\mathrm{m} / z$ : calcd for $\mathrm{C}_{11} \mathrm{H}_{14} \mathrm{IO}_{3}{ }^{+}[\mathrm{M}+\mathrm{H}]^{+}$320.9982, found 320.9987 .

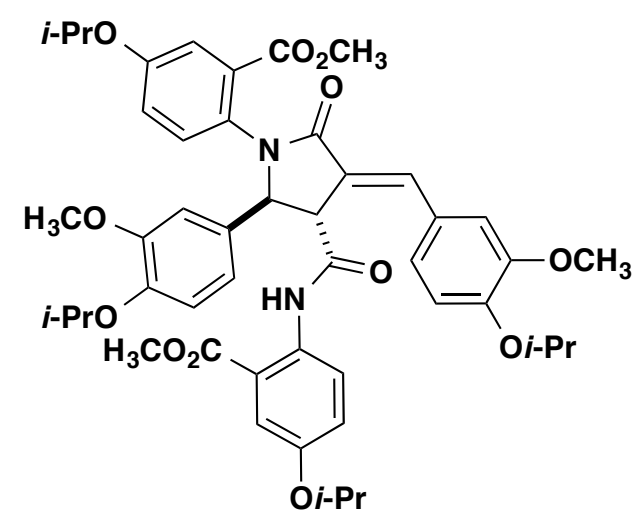

rel-methyl 5-isopropoxy-2-((2S,3S)-1-(4-isopropoxy-2-(methoxycarbonyl)phenyl)-4((E)-4-isopropoxy-3-methoxybenzylidene)-2-(4-isopropoxy-3-methoxyphenyl)-5oxopyrrolidine-3-carboxamido)benzoate (5). To a flame-dried, 7-mL vial were added aryl-iodide 6 (70. mg, $0.22 \mathrm{mmol}$ ), $\mathrm{K}_{3} \mathrm{PO}_{4}$ ( $93 \mathrm{mg}, 0.44 \mathrm{mmol}$ ), $\mathrm{CuI}$ (4.2 mg, 0.022 mmol), and bis-amide 7 (51 $\mathrm{mg}, 0.11 \mathrm{mmol})$. The vial was placed under vacuum and backfilled with argon three times. After the third fill with argon, the vial was stoppered 
and an atmosphere of argon was maintained with a needle through the septum. To a separate dry 7-mL vial were added trans-1,2-dimethylcyclohexane (6.3 mg, $0.044 \mathrm{mmol})$ and $0.55 \mathrm{~mL}$ of dioxane. The ligand solution was added to the reaction vial via syringe and the reaction was heated to $50^{\circ} \mathrm{C}$ in a pre-heated oil bath (note: oil bath temperature varied from $45-60{ }^{\circ} \mathrm{C}$ through the reaction) for 72 hours. The reaction was allowed to cool to room temperature, filtered through a pad of Celite with EtOAc $(40 \mathrm{~mL})$ and concentrated in vacuo. The residue was purified by flash column chromatography (50:50 EtOAc:hex, $\left.\mathrm{R}_{\mathrm{f}}=0.3\right)$ to yield $5(68 \mathrm{mg}, 72 \%)$ as a yellow foam: ${ }^{1} \mathrm{H}$ NMR (800 MHz, $\left.\mathrm{CDCl}_{3}\right) \delta 10.78(\mathrm{~s}, 1 \mathrm{H}), 8.59(\mathrm{~d}, J=9.2 \mathrm{~Hz}, 1 \mathrm{H}), 7.88$ (d, $\left.J=2.2 \mathrm{~Hz}, 1 \mathrm{H}\right), 7.44$ (d, $J=3.1$ $\mathrm{Hz}, 1 \mathrm{H}), 7.39$ (s, 1H), $7.12(\mathrm{dd}, J=8.5,2.1 \mathrm{~Hz}, 1 \mathrm{H}), 7.08-7.05(\mathrm{~m}, 2 \mathrm{H}), 6.89$ (d, $J=2.0$ $\mathrm{Hz}, 1 \mathrm{H}), 6.86-6.78(\mathrm{~m}, 5 \mathrm{H}), 5.24(\mathrm{~d}, J=2.8 \mathrm{~Hz}, 1 \mathrm{H}), 4.56-4.46(\mathrm{~m}, 4 \mathrm{H}), 4.26(\mathrm{dd}, J=$ 2.6, 2.6 Hz, 1H), 3.78 (d, $J=1.1 \mathrm{~Hz}, 6 \mathrm{H}), 3.77$ (s, 3H), 3.65 (s, 3H), 1.35-1.29 (m, 24H); ${ }^{13} \mathrm{C}$ NMR (201 MHz, $\left.\mathrm{CDCl}_{3}\right) \delta$ 169.0, 168.6, 167.2, 166.0, 156.8, 153.4, 150.7, 150.1, $148.9,147.4,137.2,133.7,133.4,129.5,129.2$, 127.3, 124.8, 124.4 (2), 122.6, 122.2, 119.7, 119.6, 118.4, 118.0, 117.7, 115.4, 114.4, 112.9, 110.5, 71.3, 71.2, 70.7, 70.4, 67.8, $56.14,56.12,56.0,52.6,52.4,22.2,22.17,22.1,22.08,22.07,22.0(3) ;{ }^{\star}$ IR (thin film) 2978, 2934, 1727,1694, 1645, 1600, $1503 \mathrm{~cm}^{-1}$; AMM (ESI-TOF) $\mathrm{m} / z$ : calcd for $\mathrm{C}_{48} \mathrm{H}_{57} \mathrm{~N}_{2} \mathrm{O}_{12}{ }^{+}[\mathrm{M}+\mathrm{H}]^{+}$853.3906, found 853.3914.

\$ The isopropyl methyl carbons are observed to be diastereotopic. 


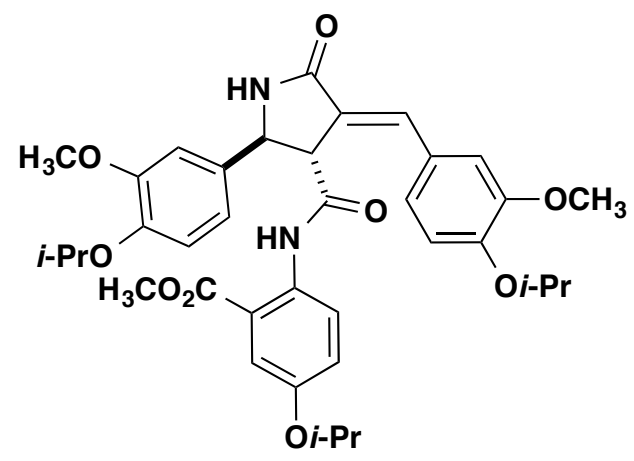

Note: If the reaction is not run to completion the mono-arylation product is observed and can be purified with the same column conditions (50:50 EtOAc:hex, $\left.R_{f}=0.15\right)$ and has the following properties:

rel-methyl 5-isopropoxy-2-((2S,3S)-4-((E)-4-isopropoxy-3-methoxybenzylidene)-2(4-isopropoxy-3-methoxyphenyl)-5-oxopyrrolidine-3-carboxamido)benzoate (16). ${ }^{1} \mathrm{H}$ NMR (600 MHz, $\left.\mathrm{CDCl}_{3}\right) \delta 11.02(\mathrm{~s}, 1 \mathrm{H}), 8.59(\mathrm{~d}, J=9.2 \mathrm{~Hz}, 1 \mathrm{H}), 7.75$ (d, $J=2.2 \mathrm{~Hz}$, 1H), 7.46 (d, $J=3.0 \mathrm{~Hz}, 1 \mathrm{H}), 7.09-7.04(\mathrm{~m}, 2 \mathrm{H}), 7.02$ (d, $J=2.1 \mathrm{~Hz}, 1 \mathrm{H}), 6.90-6.86$ (m, $3 \mathrm{H}), 6.82(\mathrm{~d}, J=8.5 \mathrm{~Hz}, 1 \mathrm{H}), 6.60(\mathrm{~d}, J=8.6 \mathrm{~Hz}, 1 \mathrm{H}), 4.98(\mathrm{~d}, J=2.2 \mathrm{~Hz}, 1 \mathrm{H}), 4.53-$ $4.49(\mathrm{~m}, 3 \mathrm{H}), 4.07$ (dd, $J=2.2,2.2 \mathrm{~Hz}, 1 \mathrm{H}), 3.85$ (s, 3H), 3.82 (s, 3H), 3.77 (s, 3H), 1.36$1.30(\mathrm{~m}, 18 \mathrm{H}) ;{ }^{13} \mathrm{C}$ NMR $\left(151 \mathrm{MHz}, \mathrm{CDCl}_{3}\right) \delta 171.3,169.5,167.6,153.4,151.0,150.1$, 149.1, 147.4, 137.3, 135.2, 133.9, 127.0, 124.4, 123.5, 122.5, 122.2, 117.7, 117.6, 117.4, $115.9,114.4,112.9,109.3,71.6,71.2,70.7,59.9,57.6,56.2,56.1,52.7,22.2,22.1,22.0$; IR (thin film) 3340, 2978, 2926, 1693, $1645 \mathrm{~cm}^{-1}$; AMM (ESI-TOF) $\mathrm{m} / \mathrm{z}$ : calcd for $\mathrm{C}_{37} \mathrm{H}_{45} \mathrm{~N}_{2} \mathrm{O}_{9}^{+}[\mathrm{M}+\mathrm{H}]^{+}$661.3120, found 661.3181 . 
Table S1. Optimization of Buchwald $N$-arylation
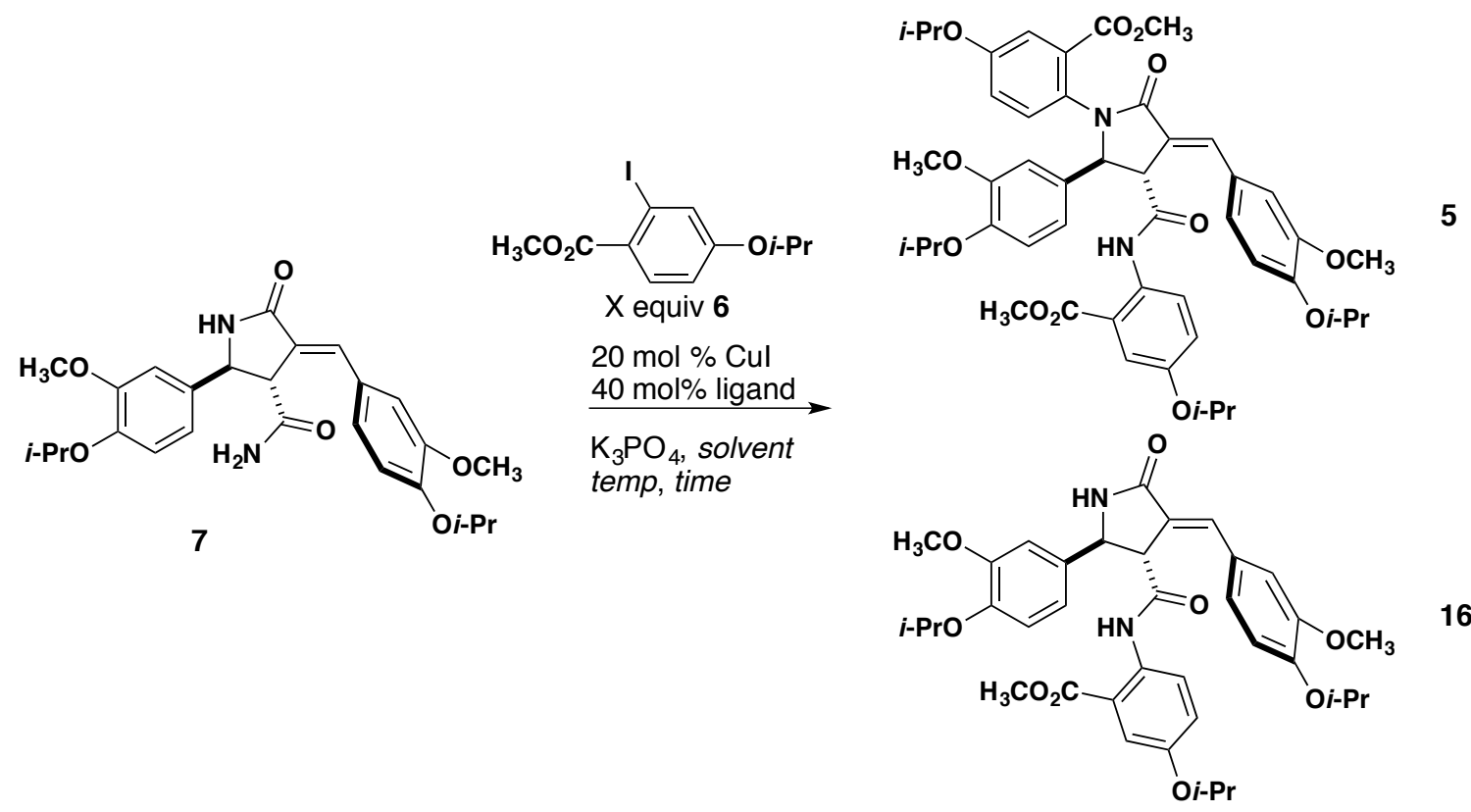

\begin{tabular}{|c|c|c|c|c|c|c|c|}
\hline entry & ligand & $\begin{array}{l}\text { equiv. } \\
\text { (6) }\end{array}$ & solvent & $\begin{array}{l}\text { temp } \\
\left({ }^{\circ} \mathrm{C}\right)\end{array}$ & $\begin{array}{l}\text { time } \\
\text { (h) }\end{array}$ & $\begin{array}{c}\text { yield } 5 \\
(\%)\end{array}$ & $\begin{array}{c}\text { yield } 16 \\
(\%)\end{array}$ \\
\hline $\begin{array}{l}1 \\
2 \\
3 \\
4 \\
5 \\
6 \\
7 \\
8 \\
9\end{array}$ & $\begin{array}{c}\text { DMEDA } \\
\text { DMEDA } \\
\text { DMEDA } \\
\text { DMEDA } \\
\text { DMEDA } \\
\text { DMPAO } \\
\text { A } \\
\mathbf{A} \\
\mathbf{A}\end{array}$ & $\begin{array}{l}2 \\
2 \\
2 \\
2 \\
2 \\
2 \\
2 \\
3 \\
4\end{array}$ & $\begin{array}{c}\text { DMF } \\
\text { DMSO } \\
\text { dioxane } \\
\text { dioxane } \\
\text { dioxane } \\
\text { dioxane } \\
\text { dioxane } \\
\text { dioxane } \\
\text { dioxane }\end{array}$ & $\begin{array}{l}100 \\
100 \\
70 \\
50 \\
50 \\
50 \\
50 \\
50 \\
50\end{array}$ & $\begin{array}{l}24 \\
24 \\
24 \\
24 \\
48 \\
48 \\
48 \\
48 \\
72\end{array}$ & $\begin{array}{c}\text { dec. } \\
\text { dec. } \\
\text { trace } \\
13 \\
22 \\
\text { dec }^{a} \\
55 \\
55 \\
72\end{array}$ & $\begin{array}{c}\text { dec. } \\
\text { dec. } \\
\text { nd } \\
44 \\
40 \\
\operatorname{dec}^{\mathrm{a}} \\
\mathrm{nd} \mathrm{d}^{\mathrm{b}} \\
\mathrm{nd} \\
\mathrm{nd}^{\mathrm{b}}\end{array}$ \\
\hline \multicolumn{8}{|c|}{ a. decomposed, b. not detected. } \\
\hline & DMPAO & & & 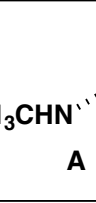 & & & \\
\hline
\end{tabular}




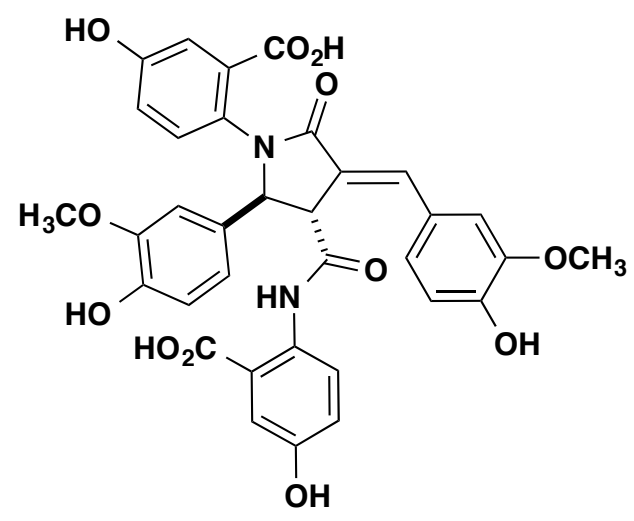

rel-2-((2S,3S)-1-(2-carboxy-4-hydroxyphenyl)-4-((E)-4-hydroxy-3methoxybenzylidene)-2-(4-hyd roxy-3-methoxyphenyl)-5-oxopyrrolidine-3carboxamido)-5-hydroxybenzoic acid; Bisavenanthramide B-6 (2). A solution of 5 (52 mg, $0.061 \mathrm{mmol}$ ) in $0.5 \mathrm{~mL}$ of $\mathrm{CH}_{2} \mathrm{Cl}_{2}$ was transferred to a dry $7-\mathrm{mL}$ vial via syringe. The solution was cooled to $0{ }^{\circ} \mathrm{C}$ and $1.0 \mathrm{M} \mathrm{BCl}_{3}$ in $\mathrm{CH}_{2} \mathrm{Cl}_{2}(0.73 \mathrm{~mL}, 0.73 \mathrm{mmol})$ was added slowly to the reaction over 3 minutes. The reaction was slowly warmed to room temperature by allowing the ice bath to melt over one hour. After one hour, the ice bath was removed and the reaction was stirred for 2 hours at room temperature. The reaction was quenched with water. The layers were separated and the aqueous layer was extracted with EtOAc $(3 \times 5 \mathrm{~mL})$. The combined organic layers were dried over $\mathrm{Na}_{2} \mathrm{SO}_{4}$, gravity filtered, and concentrated in vacuo. The crude material was dissolved in $1.0 \mathrm{~mL}$ of water and $1.0 \mathrm{~mL}$ of THF. LiOH (30 mg, $1.25 \mathrm{mmol})$ was added to the reaction. The reaction was stirred for 17 hours. The reaction was acidified to $\mathrm{pH} 2$ with $3 \mathrm{M} \mathrm{HCl}$ and extracted with EtOAc $(3 \times 5 \mathrm{~mL})$. The combined organic layers were dried over $\mathrm{Na}_{2} \mathrm{SO}_{4}$, gravity filtered, and concentrated in vacuo to provide Bisavenanthramide B-6 (2) (31 mg, 78\%) as an orange solid: ${ }^{1} \mathrm{H}$ NMR $\left(600 \mathrm{MHz}, \mathrm{CD}_{3} \mathrm{OD}\right) \delta 8.24(\mathrm{~d}, J=9.0 \mathrm{~Hz}, 1 \mathrm{H}), 7.70(\mathrm{~d}, J=$ $2.4 \mathrm{~Hz}, 1 \mathrm{H}), 7.38$ (d, $J=3.0 \mathrm{~Hz}, 1 \mathrm{H}), 7.37$ (d, $J=2.6 \mathrm{~Hz}, 1 \mathrm{H}), 7.08$ (d, $J=1.9 \mathrm{~Hz}, 1 \mathrm{H}$ ), 7.04 (dd, $J=8.2,2.0 \mathrm{~Hz}, 1 \mathrm{H}), 6.99$ (s, 1H), $6.95(\mathrm{dd}, J=9.0,3.0 \mathrm{~Hz}, 1 \mathrm{H}), 6.88-6.76(\mathrm{~m}$, $4 \mathrm{H}), 6.73(\mathrm{~d}, J=8.1 \mathrm{~Hz}, 1 \mathrm{H}), 5.33(\mathrm{~d}, J=3.6 \mathrm{~Hz}, 1 \mathrm{H}), 4.28(\mathrm{dd}, J=3.8,2.4 \mathrm{~Hz}, 1 \mathrm{H})$, $3.76(\mathrm{~s}, 3 \mathrm{H}), 3.73$ (s, 3H);* ${ }^{13} \mathrm{C}$ NMR (151 MHz, CD $\left.{ }_{3} \mathrm{OD}\right) \delta 170.7$ (2), 170.1, 168.4, $157.9,154.5,149.7,149.4,149.0,147.9,138.2,133.6,132.8,131.1$ (2), 129.5, 127.6, 126.3, 125.9, 123.8, 121.7, 121.6, 120.4, 120.1, 118.8, 118.0, 116.5, 116.3, 113.5, 111.8, 
69.2, 57.1, 56.4, 56.3; IR (thin film) 3198 (broad), 2926, 1860, $1603 \mathrm{~cm}^{-1}$; AMM (ESITOF) $m / z$ : calcd for $\mathrm{C}_{34} \mathrm{H}_{27} \mathrm{~N}_{2} \mathrm{O}_{12}{ }^{-}[\mathrm{M}-\mathrm{H}]^{-} 655.1569$, found 655.1577.

*The carboxylic acid, phenol, and amide protons are not observed.

\section{References}

1. Parker, L. L.; Gowans, N. D.; Jones, S. W.; Robins, D. J., Tetrahedron 2003, 59, 10165-10171.

2. Younai, A.; Chin, G. F.; Fettinger, J. C.; Shaw, J. T., J. Org. Chem. 2010, 75, $8333-8336$.

3. Miura, T.; Nakashima, K.; Tada, N.; Itoh, A., Chem. Commun. 2011, 47, 18751877. 


\section{X-ray Crystal Structure of 17}

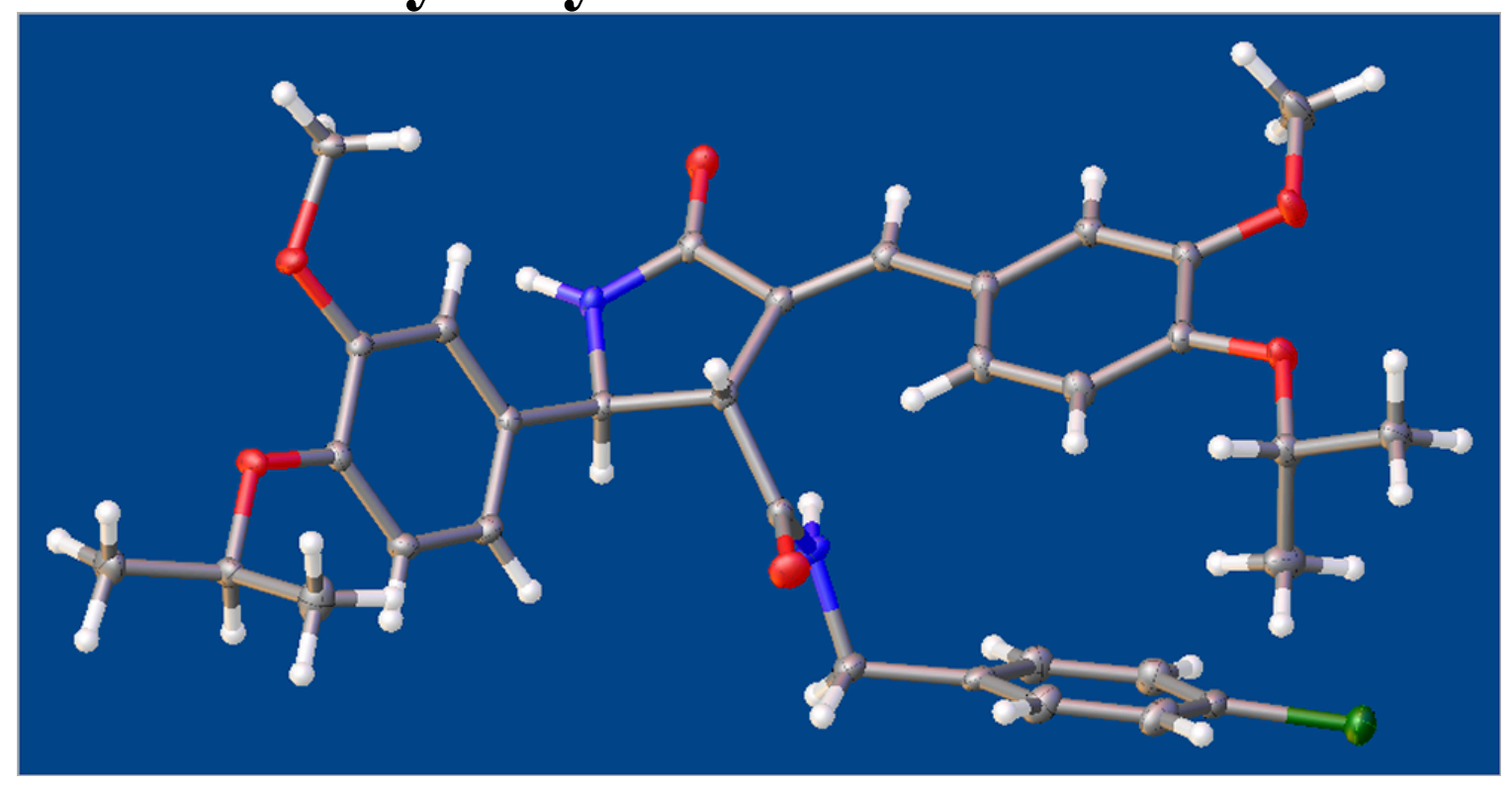

Table 1 Crystal data and structure refinement for MJD010_0m.

Identification code

MJD010_0m

Empirical formula

$\mathrm{C}_{33} \mathrm{H}_{37} \mathrm{~N}_{2} \mathrm{O}_{6} \mathrm{Cl}$

Formula weight

555.80

Temperature/K

296.15

Crystal system

monoclinic

Space group

$\mathrm{C} 2 / \mathrm{c}$

$\mathrm{a} / \AA ̊$

$25.162(5)$

$\mathrm{b} / \AA$

15.406(3)

c/Å

16.174(4)

$\alpha{ }^{\circ}$

90

$\beta /{ }^{\circ}$

109.269(3)

$\gamma /{ }^{\circ}$

90

Volume $/ \AA^{3}$

$5919(2)$

$\mathrm{Z}$

8

$\varrho_{\text {calc }} \mathrm{g} / \mathrm{cm}^{3}$

1.247

$\mu / \mathrm{mm}^{-1}$

0.175

$\mathrm{F}(000)$

2216.0

Crystal size $/ \mathrm{mm}^{3}$

$0.364 \times 0.279 \times 0.252$

Radiation

$\operatorname{MoK} \alpha(\lambda=0.71073)$

$2 \Theta$ range for data collection $/{ }^{\circ} 3.15$ to 55.26

Index ranges

$-32 \leq \mathrm{h} \leq 32,-20 \leq \mathrm{k} \leq 19,-21 \leq 1 \leq 20$

Reflections collected

38353

Independent reflections

$6868\left[R_{\text {int }}=0.0202, R_{\text {sigma }}=0.0133\right]$

Data/restraints/parameters

$6868 / 0 / 385$

Goodness-of-fit on $\mathrm{F}^{2}$

1.047

Final $R$ indexes $[I>=2 \sigma(I)] \quad R_{1}=0.0350, \mathrm{wR}_{2}=0.0932$

Final $\mathrm{R}$ indexes [all data] $\quad \mathrm{R}_{1}=0.0371, \mathrm{wR}_{2}=0.0953$

Largest diff. peak/hole / e $\AA^{-3} 0.42 /-0.50$ 
Table 2 Fractional Atomic Coordinates $\left(\times 10^{4}\right)$ and Equivalent Isotropic Displacement Parameters $\left(\AA^{2} \times 10^{3}\right)$ for MJD010_0m. $U_{e q}$ is defined as $1 / 3$ of of the trace of the orthogonalised $U_{\mathrm{IJ}}$ tensor.

\begin{tabular}{|c|c|c|c|c|}
\hline Atom & $x$ & $y$ & $z$ & $\mathrm{U}(\mathbf{e q})$ \\
\hline $\mathrm{Cl1}$ & $7163.5(2)$ & $7111.3(2)$ & $2552.3(2)$ & $27.59(9)$ \\
\hline $\mathrm{O} 4$ & $6242.9(3)$ & $-79.4(5)$ & $7473.1(5)$ & $15.63(16)$ \\
\hline $\mathrm{O} 3$ & $5184.5(3)$ & $179.6(5)$ & $6617.1(5)$ & $15.59(16)$ \\
\hline $\mathrm{O} 1$ & $4325.8(3)$ & $2519.4(5)$ & $2823.1(5)$ & $19.24(17)$ \\
\hline $\mathrm{O} 6$ & $5747.8(3)$ & $7393.9(5)$ & $4862.9(5)$ & $18.92(17)$ \\
\hline $\mathrm{O} 2$ & $6613.0(3)$ & $3454.6(6)$ & $5211.6(5)$ & $21.54(17)$ \\
\hline O5 & $4860.6(3)$ & $7251.9(5)$ & $3497.7(6)$ & $22.69(18)$ \\
\hline N2 & $6314.7(4)$ & $3278.5(6)$ & $3734.7(6)$ & $16.71(18)$ \\
\hline N1 & $5070.6(4)$ & $1774.0(6)$ & $3754.2(6)$ & $14.21(17)$ \\
\hline $\mathrm{C} 7$ & $5556.2(4)$ & $552.1(6)$ & $6262.3(7)$ & $12.76(19)$ \\
\hline C6 & $5396.0(4)$ & $1036.4(6)$ & $5495.4(6)$ & $13.34(19)$ \\
\hline $\mathrm{C} 8$ & $6131.2(4)$ & $410.4(6)$ & $6728.8(6)$ & $13.11(19)$ \\
\hline $\mathrm{C} 5$ & $5803.4(4)$ & $1376.2(6)$ & $5169.9(6)$ & $13.13(19)$ \\
\hline C9 & $6533.3(4)$ & $758.7(7)$ & $6406.2(7)$ & $15.5(2)$ \\
\hline $\mathrm{C} 1$ & $4794.2(4)$ & $2501.0(7)$ & $3393.6(7)$ & $14.1(2)$ \\
\hline $\mathrm{C} 18$ & $6243.9(4)$ & $3260.2(7)$ & $4522.4(7)$ & $14.6(2)$ \\
\hline $\mathrm{C} 2$ & $5144.8(4)$ & $3260.0(7)$ & $3820.7(6)$ & $13.31(19)$ \\
\hline $\mathrm{C} 10$ & $6368.6(4)$ & $1233.4(7)$ & $5627.7(7)$ & $15.5(2)$ \\
\hline C14 & $5097.5(4)$ & $6483.4(7)$ & $3856.1(7)$ & $16.1(2)$ \\
\hline $\mathrm{C} 4$ & $5644.3(4)$ & $1912.3(7)$ & $4341.1(6)$ & $12.97(19)$ \\
\hline $\mathrm{C} 20$ & $6918.9(4)$ & $4372.9(7)$ & $3361.2(7)$ & $16.2(2)$ \\
\hline $\mathrm{C} 3$ & $5668.6(4)$ & $2917.8(6)$ & $4514.2(6)$ & $12.86(19)$ \\
\hline $\mathrm{C} 13$ & $4900.9(4)$ & $5666.4(7)$ & $3542.7(7)$ & $16.0(2)$ \\
\hline $\mathrm{C} 16$ & $5851.7(5)$ & $5817.9(7)$ & $5014.4(7)$ & $18.3(2)$ \\
\hline $\mathrm{C} 27$ & $6824.9(4)$ & $-220.6(7)$ & $7992.8(7)$ & $15.6(2)$ \\
\hline $\mathrm{C} 15$ & $5585.2(4)$ & $6565.1(7)$ & $4601.0(7)$ & $15.6(2)$ \\
\hline $\mathrm{C} 17$ & $5649.7(5)$ & $5001.4(7)$ & $4703.2(7)$ & $17.6(2)$ \\
\hline C11 & $4946.5(4)$ & $4066.8(7)$ & $3596.5(7)$ & $14.7(2)$ \\
\hline $\mathrm{C} 12$ & $5178.6(4)$ & $4907.2(7)$ & $3955.6(7)$ & $14.9(2)$ \\
\hline $\mathrm{C} 21$ & $6746.0(5)$ & 4607.6 ( 8 ) & $2481.6(7)$ & $20.9(2)$ \\
\hline $\mathrm{C} 23$ & $7053.5(5)$ & $6061.0(7)$ & $2864.7(8)$ & $19.0(2)$ \\
\hline C19 & $6859.2(5)$ & $3447.5(7)$ & $3632.3(8)$ & $19.5(2)$ \\
\hline $\mathrm{C} 26$ & $4614.7(4)$ & $498.8(7)$ & $6299.0(7)$ & $17.3(2)$ \\
\hline C31 & $6293.5(4)$ & $7516.1(7)$ & $5524.1(7)$ & $17.2(2)$ \\
\hline C33 & $6309.1(5)$ & $8451.8(7)$ & $5820.9(8)$ & $19.6(2)$ \\
\hline $\mathrm{C} 24$ & $7224.9(5)$ & $5849.4(8)$ & $3746.7(8)$ & $22.2(2)$ \\
\hline $\mathrm{C} 25$ & $7154.7(5)$ & $5003.4(8)$ & $3990.5(7)$ & $21.5(2)$ \\
\hline $\mathrm{C} 22$ & $6808.2(5)$ & $5452.6(8)$ & $2221.3(8)$ & $22.1(2)$ \\
\hline $\mathrm{C} 28$ & $6829.0(5)$ & -1011.8 ( 8 ) & $8549.0(8)$ & $22.1(2)$ \\
\hline $\mathrm{C} 32$ & $6756.2(5)$ & 7309.8 ( 8 ) & $5145.2(9)$ & $26.9(3)$ \\
\hline $\mathrm{C} 30$ & $4310.7(5)$ & 7214.6 ( 8) & $2860.1(9)$ & $26.0(3)$ \\
\hline $\mathrm{C} 29$ & $7064.4(6)$ & $574.2(8)$ & $8545.3(8)$ & $25.6(3)$ \\
\hline
\end{tabular}


Table 3 Anisotropic Displacement Parameters $\left(\AA^{2} \times 10^{3}\right)$ for MJD010_0m. The Anisotropic displacement factor exponent takes the form: $-2 \pi^{2}\left[h^{2} a^{* 2} U_{11}+2 h k a * b * U_{12}+\ldots\right]$.

\begin{tabular}{|c|c|c|c|c|c|c|}
\hline Atom & $\mathrm{U}_{11}$ & $\mathbf{U}_{22}$ & $\mathbf{U}_{33}$ & & $\mathbf{U}_{13}$ & $\mathbf{U}_{12}$ \\
\hline $\mathrm{Cl1}$ & $23.15(15)$ & $16.99(14)$ & $40.96(18)$ & $4.41(11)$ & $8.31(12)$ & $-2.05(10)$ \\
\hline $\mathrm{O} 4$ & $13.1(3)$ & $18.4(4)$ & $15.1(3)$ & $5.5(3)$ & $4.2(3)$ & $2.4(3)$ \\
\hline $\mathrm{O} 3$ & $12.1(3)$ & $18.3(4)$ & $17.2(3)$ & $4.9(3)$ & $5.9(3)$ & $0.3(3)$ \\
\hline $\mathrm{O} 1$ & $17.0(4)$ & $17.9(4)$ & $18.1(4)$ & $3.3(3)$ & $-0.6(3)$ & $-3.1(3)$ \\
\hline O6 & $16.3(4)$ & $12.0(4)$ & $23.4(4)$ & $-2.7(3)$ & $-0.3(3)$ & $-0.2(3)$ \\
\hline $\mathrm{O} 2$ & $17.8(4)$ & $25.2(4)$ & $17.2(4)$ & $-3.0(3)$ & $-0.1(3)$ & $-4.1(3)$ \\
\hline O5 & $17.8(4)$ & $12.3(4)$ & $30.0(4)$ & $0.5(3)$ & $-2.8(3)$ & $1.8(3)$ \\
\hline N2 & $13.6(4)$ & $20.0(4)$ & $15.7(4)$ & $-1.1(3)$ & $3.8(3)$ & $-5.9(3)$ \\
\hline N1 & $15.1(4)$ & $11.4(4)$ & $14.2(4)$ & $0.6(3)$ & $2.2(3)$ & $-2.6(3)$ \\
\hline $\mathrm{C} 7$ & $13.5(5)$ & $11.5(4)$ & $14.5(4)$ & $-0.7(4)$ & $6.2(4)$ & $-0.6(3)$ \\
\hline C6 & $12.5(4)$ & $12.8(4)$ & $14.3(4)$ & $0.2(4)$ & $3.8(4)$ & $0.5(4)$ \\
\hline $\mathrm{C} 8$ & $15.3(5)$ & $11.5(4)$ & $12.5(4)$ & $0.6(4)$ & $4.5(4)$ & $1.7(4)$ \\
\hline $\mathrm{C} 5$ & $15.3(5)$ & $11.0(4)$ & $13.2(4)$ & $0.4(3)$ & $4.8(4)$ & $0.2(4)$ \\
\hline C9 & $12.3(4)$ & $17.6(5)$ & $16.1(5)$ & $1.5(4)$ & $4.0(4)$ & $1.3(4)$ \\
\hline $\mathrm{C} 1$ & $15.7(5)$ & $14.4(5)$ & $12.6(4)$ & $1.1(4)$ & $5.2(4)$ & $-1.8(4)$ \\
\hline $\mathrm{C} 18$ & $14.2(5)$ & $11.4(4)$ & $16.3(5)$ & $0.5(4)$ & $2.6(4)$ & $-0.7(4)$ \\
\hline $\mathrm{C} 2$ & $12.9(4)$ & $14.6(5)$ & $12.5(4)$ & $-0.1(4)$ & $4.2(4)$ & $-2.0(4)$ \\
\hline $\mathrm{C} 10$ & $14.4(5)$ & $17.0(5)$ & $16.5(5)$ & $1.7(4)$ & $6.7(4)$ & $-1.2(4)$ \\
\hline $\mathrm{C} 14$ & $14.6(5)$ & $13.4(5)$ & $19.9(5)$ & $1.2(4)$ & $5.1(4)$ & $1.7(4)$ \\
\hline $\mathrm{C} 4$ & $13.6(5)$ & $12.7(4)$ & $12.7(4)$ & $0.6(4)$ & $4.5(4)$ & $-0.8(4)$ \\
\hline $\mathrm{C} 20$ & $10.8(4)$ & $18.4(5)$ & $20.7(5)$ & $-1.6(4)$ & $6.8(4)$ & $-2.7(4)$ \\
\hline $\mathrm{C} 3$ & $14.4(5)$ & $12.2(5)$ & $11.8(4)$ & $0.3(3)$ & $4.1(4)$ & $-0.9(4)$ \\
\hline $\mathrm{C} 13$ & $13.6(5)$ & $14.9(5)$ & $17.9(5)$ & $-0.3(4)$ & $3.2(4)$ & $0.1(4)$ \\
\hline $\mathrm{C} 16$ & $19.8(5)$ & $16.5(5)$ & $15.3(5)$ & $-1.2(4)$ & $1.2(4)$ & $0.8(4)$ \\
\hline $\mathrm{C} 27$ & $13.0(5)$ & $17.0(5)$ & $14.9(5)$ & $3.3(4)$ & $2.2(4)$ & $1.4(4)$ \\
\hline $\mathrm{C} 15$ & $16.4(5)$ & $12.6(5)$ & $18.1(5)$ & $-2.1(4)$ & $6.1(4)$ & $-0.8(4)$ \\
\hline $\mathrm{C} 17$ & $22.0(5)$ & $13.4(5)$ & $15.9(5)$ & $1.2(4)$ & $4.1(4)$ & $2.1(4)$ \\
\hline $\mathrm{C} 11$ & $14.2(5)$ & $15.0(5)$ & $14.5(4)$ & $0.1(4)$ & $4.2(4)$ & $-1.0(4)$ \\
\hline $\mathrm{C} 12$ & $16.6(5)$ & $13.1(5)$ & $16.4(5)$ & $-0.1(4)$ & 7.1 ( 4 ) & $-0.3(4)$ \\
\hline $\mathrm{C} 21$ & $20.3(5)$ & $20.2(5)$ & $19.4(5)$ & $-4.1(4)$ & $2.7(4)$ & $-4.4(4)$ \\
\hline $\mathrm{C} 23$ & $14.0(5)$ & $15.0(5)$ & $29.3(6)$ & $0.4(4)$ & $8.9(4)$ & $-0.8(4)$ \\
\hline $\mathrm{C} 19$ & $15.5(5)$ & $18.5(5)$ & $26.0(5)$ & $0.6(4)$ & $8.9(4)$ & $-2.1(4)$ \\
\hline $\mathrm{C} 26$ & $11.8(5)$ & $22.1(5)$ & $18.6(5)$ & $2.4(4)$ & $5.8(4)$ & $1.0(4)$ \\
\hline $\mathrm{C} 31$ & $15.8(5)$ & $14.4(5)$ & $18.2(5)$ & $-2.0(4)$ & $1.1(4)$ & $-0.2(4)$ \\
\hline $\mathrm{C} 33$ & $17.8(5)$ & $14.6(5)$ & $24.2(5)$ & $-4.0(4)$ & $3.9(4)$ & $-0.9(4)$ \\
\hline $\mathrm{C} 24$ & $23.0(5)$ & $21.4(6)$ & $24.8(6)$ & $-8.4(4)$ & 11.1 ( 4 ) & $-7.6(4)$ \\
\hline $\mathrm{C} 25$ & $23.6(6)$ & $24.9(6)$ & $17.6(5)$ & $-3.5(4)$ & $9.0(4)$ & $-6.9(4)$ \\
\hline $\mathrm{C} 22$ & $20.1(5)$ & $23.2(6)$ & $20.0(5)$ & $1.8(4)$ & $2.4(4)$ & $-1.7(4)$ \\
\hline $\mathrm{C} 28$ & $21.1(5)$ & $19.9(5)$ & $22.3(5)$ & $7.8(4)$ & $3.0(4)$ & $1.3(4)$ \\
\hline C32 & $20.8(6)$ & $23.5(6)$ & $37.0(7)$ & $-10.1(5)$ & $10.3(5)$ & $-1.9(5)$ \\
\hline $\mathrm{C} 30$ & $19.5(6)$ & $17.5(5)$ & $31.8(6)$ & $-1.2(5)$ & $-4.1(5)$ & $4.3(4)$ \\
\hline $\mathrm{C} 29$ & $31.7(6)$ & $22.1(6)$ & $18.5(5)$ & $0.1(4)$ & $2.2(5)$ & $-6.5(5)$ \\
\hline
\end{tabular}


Table 4 Bond Lengths for MJD010_0m.

\begin{tabular}{|c|c|c|c|c|c|}
\hline \multicolumn{2}{|c|}{ Atom Atom } & \multirow{2}{*}{$\begin{array}{c}\text { Length/Å } \\
1.7443(12)\end{array}$} & \multicolumn{2}{|c|}{ Atom Atom } & \multirow{2}{*}{$\begin{array}{c}\text { Length/Å } \\
1.5366(14)\end{array}$} \\
\hline C11 & $\mathrm{C} 23$ & & $\mathrm{C} 18$ & $\mathrm{C} 3$ & \\
\hline & & 12 ) & & & \\
\hline & & & & & \\
\hline & & & $\mathrm{C} 14$ & & \\
\hline & & & & & \\
\hline & & $3)$ & $\mathrm{C} 4$ & & \\
\hline & & $3)$ & $\mathrm{C} 20$ & & 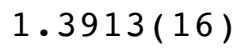 \\
\hline & & 3 ) & $\mathrm{C} 20$ & & \\
\hline & $\mathrm{C} 18$ & 1.22 & $\mathrm{C} 20$ & & 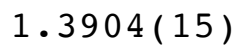 \\
\hline & & & 13 & & \\
\hline & ( & 4) & $\mathrm{C} 16$ & & 5 \\
\hline & ( & 1.3 & $\mathrm{C} 16$ & & \\
\hline 2 & & 1.4 & $\mathrm{C} 27$ & $\mathrm{C}$ & \\
\hline & & 4) & $\mathrm{C} 27$ & C & 1.5 \\
\hline & $\mathrm{C} 4$ & 1.4 & $\mathrm{C} 17$ & & 1.3 \\
\hline & & 1.3 & $\mathrm{C} 11$ & $\mathrm{C}$ & 1.4 \\
\hline & $\mathrm{C} 8$ & 1.4 & $\mathrm{C} 21$ & $\mathrm{C} 2$ & 1.3 \\
\hline & & 1.3 & $\mathrm{C} 23$ & $\mathrm{C} 24$ & 1.3 \\
\hline & & 1.39 & $\mathrm{C} 23$ & & 1.3 \\
\hline & $\mathrm{C} 1$ & 1.38 & $\mathrm{C} 31$ & $\mathrm{C} 33$ & 1.5 \\
\hline & $\mathrm{C} 4$ & 1.51 & $\mathrm{C} 31$ & $\mathrm{C}$ & 1.5 \\
\hline & $\mathrm{C} 1 \mathrm{C}$ & 1.39 & $\mathrm{C} 24$ & & \\
\hline 1 & $\mathrm{C} 2$ & $1.4901(14)$ & & & \\
\hline
\end{tabular}

Table 5 Bond Angles for MJD010_0m.

\begin{tabular}{llllllll} 
Atom Atom Atom & \multicolumn{1}{l}{ Angle $^{\circ}$} & \multicolumn{3}{c}{ Atom Atom Atom } & Angle $^{\circ}$ \\
C8 & O4 & C27 & $117.99(8)$ & C5 & C4 & C3 & $113.40(8)$ \\
C7 & O3 & C26 & $116.27(8)$ & C21 & C20 & C19 & $120.90(10)$ \\
C15 & O6 & C31 & $117.72(8)$ & C25 & C20 & C21 & $118.74(10)$ \\
C14 & O5 & C30 & $117.06(9)$ & C25 & C20 & C19 & $120.35(10)$ \\
C18 & N2 & C19 & $122.38(9)$ & C18 & C3 & C4 & $108.72(8)$ \\
C1 & N1 & C4 & $114.82(8)$ & C2 & C3 & C18 & $118.28(8)$ \\
O3 & C7 & C6 & $124.12(9)$ & C2 & C3 & C4 & $103.75(8)$ \\
O3 & C7 & C8 & $115.55(9)$ & C14 & C13 & C12 & $121.20(10)$ \\
C6 & C7 & C8 & $120.33(9)$ & C17 & C16 & C15 & $121.09(10)$ \\
C7 & C6 & C5 & $120.30(9)$ & O4 & C27 & C28 & $105.90(8)$ \\
O4 & C8 & C7 & $115.62(9)$ & O4 & C27 & C29 & $110.51(9)$ \\
O4 & C8 & C9 & $125.39(9)$ & C28 & C27 & C29 & $112.10(9)$ \\
C9 & C8 & C7 & $118.99(9)$ & O6 & C15 & C14 & $115.78(9)$ \\
C6 & C5 & C4 & $121.71(9)$ & O6 & C15 & C16 & $125.36(10)$ \\
C10 & C5 & C6 & $119.28(9)$ & C16 & C15 & C14 & $118.84(10)$
\end{tabular}




$\begin{array}{lllrlllr}\mathrm{C} 10 & \mathrm{C} 5 & \mathrm{C} 4 & 119.00(9) & \mathrm{C} 16 & \mathrm{C} 17 & \mathrm{C} 12 & 120.93(10) \\ \mathrm{C} 8 & \mathrm{C} 9 & \mathrm{C} 10 & 120.31(9) & \mathrm{C} 2 & \mathrm{C} 11 & \mathrm{C} 12 & 130.45(10) \\ \mathrm{O} 1 & \mathrm{C} 1 & \mathrm{~N} 1 & 124.94(10) & \mathrm{C} 13 & \mathrm{C} 12 & \mathrm{C} 11 & 118.41(9) \\ \mathrm{O} 1 & \mathrm{C} 1 & \mathrm{C} 2 & 126.99(10) & \mathrm{C} 17 & \mathrm{C} 12 & \mathrm{C} 13 & 118.07(10) \\ \mathrm{N} 1 & \mathrm{C} 1 & \mathrm{C} 2 & 108.06(9) & \mathrm{C} 17 & \mathrm{C} 12 & \mathrm{C} 11 & 123.48(10) \\ \mathrm{O} 2 & \mathrm{C} 18 & \mathrm{~N} 2 & 123.89(10) & \mathrm{C} 20 & \mathrm{C} 21 & \mathrm{C} 22 & 121.57(10) \\ \mathrm{O} 2 & \mathrm{C} 18 & \mathrm{C} 3 & 121.28(10) & \mathrm{C} 24 & \mathrm{C} 23 & \mathrm{C} 11 & 119.42(9) \\ \mathrm{N} 2 & \mathrm{C} 18 & \mathrm{C} 3 & 114.73(9) & \mathrm{C} 22 & \mathrm{C} 23 & \mathrm{C} 11 & 118.95(9) \\ \mathrm{C} 1 & \mathrm{C} 2 & \mathrm{C} 3 & 107.90(9) & \mathrm{C} 22 & \mathrm{C} 23 & \mathrm{C} 24 & 121.62(11) \\ \mathrm{C} 11 & \mathrm{C} 2 & \mathrm{C} 1 & 119.37(9) & \mathrm{N} 2 & \mathrm{C} 19 & \mathrm{C} 20 & 112.85(9) \\ \mathrm{C} 11 & \mathrm{C} 2 & \mathrm{C} 3 & 132.54(9) & \mathrm{O} 6 & \mathrm{C} 31 & \mathrm{C} 33 & 106.07(8) \\ \mathrm{C} 5 & \mathrm{C} 10 & \mathrm{C} 9 & 120.77(9) & \mathrm{O} 6 & \mathrm{C} 31 & \mathrm{C} 32 & 109.97(9) \\ \mathrm{O} 5 & \mathrm{C} 14 & \mathrm{C} 13 & 125.37(10) & \mathrm{C} 33 & \mathrm{C} 31 & \mathrm{C} 32 & 112.58(9) \\ \mathrm{O} 5 & \mathrm{C} 14 & \mathrm{C} 15 & 114.79(9) & \mathrm{C} 23 & \mathrm{C} 24 & \mathrm{C} 25 & 119.15(10) \\ \mathrm{C} 13 & \mathrm{C} 14 & \mathrm{C} 15 & 119.84(10) & \mathrm{C} 24 & \mathrm{C} 25 & \mathrm{C} 20 & 120.73(11) \\ \mathrm{N} 1 & \mathrm{C} 4 & \mathrm{C} 5 & 114.40(8) & \mathrm{C} 23 & \mathrm{C} 22 & \mathrm{C} 21 & 118.17(11) \\ \mathrm{N} 1 & \mathrm{C} 4 & \mathrm{C} 3 & 103.41(8) & & & & \end{array}$

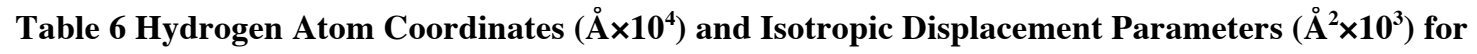
MJD010_0m.

\begin{tabular}{lrrrr} 
Atom & \multicolumn{3}{c}{$\boldsymbol{z}$} & $\mathbf{U}(\mathbf{e q})$ \\
H2 & 6028 & $\mathbf{y}$ & 3274 & 20 \\
H1 & 4921 & 1267 & 3647 & 17 \\
H6 & 5016 & 1135 & 5197 & 16 \\
H9 & 6914 & 675 & 6711 & 19 \\
H10 & 1457 & 5413 & 19 \\
H4 & 6641 & 1772 & 4024 & 16 \\
H3 & 5905 & 3021 & 5092 & 19 \\
H13 & 5633 & 5617 & 3051 & 22 \\
H16 & 4580 & 5865 & 5508 & 19 \\
H27 & 6171 & -342 & 7605 & 21 \\
H17 & 7044 & 4510 & 4998 & 18 \\
H11 & 5831 & 4094 & 3138 & 23 \\
H21 & 4606 & 4190 & 2057 & 23 \\
H19A & 6585 & 3055 & 3195 & 26 \\
H19B & 6910 & 3328 & 4182 & 26 \\
H26A & 7154 & 311 & 5706 & 26 \\
H26B & 4437 & 1122 & 6320 & 21 \\
H26C & 4618 & 277 & 6659 & 29 \\
H31 & 4409 & 7130 & 6021 & 29 \\
H33A & 6328 & 8831 & 5340 & 29 \\
H33B & 6289 & 8557 & 6292 & 27 \\
H33C & 6654 & 8561 & 6019 & 26 \\
H24 & 5995 & 6268 & 4170 &
\end{tabular}




$\begin{array}{lrrrr}\text { H22 } & 6688 & 5604 & 1632 & 27 \\ \text { H28A } & 6641 & -878 & 8963 & 33 \\ \text { H28B } & 7211 & -1178 & 8857 & 33 \\ \text { H28C } & 6637 & -1481 & 8180 & 33 \\ \text { H32A } & 6702 & 6734 & 4904 & 40 \\ \text { H32B } & 7115 & 7344 & 5600 & 40 \\ \text { H32C } & 6744 & 7720 & 4692 & 40 \\ \text { H30A } & 4328 & 6919 & 2347 & 39 \\ \text { H30B } & 4172 & 7793 & 2706 & 39 \\ \text { H30C } & 4063 & 6907 & 3098 & 39 \\ \text { H29A } & 7031 & 1069 & 8171 & 38 \\ \text { H29B } & 7454 & 477 & 8872 & 38 \\ \text { H29C } & 6860 & 679 & 8942 & 38\end{array}$

\section{Experimental}

Single crystals of $\mathrm{C}_{33} \mathrm{H}_{37} \mathrm{~N}_{2} \mathrm{O}_{6} \mathrm{Cl}$ were grown from a supersaturated solution in methanol. A suitable crystal was selected and mounted on a 'Bruker APEX-II CCD' diffractometer. The crystal was kept at 296.15 K during data collection. Using Olex2 [1], the structure was solved with the XT [2] structure solution program using Direct Methods and refined with the XL [3] refinement package using Least Squares minimisation.

1. Dolomanov, O.V., Bourhis, L.J., Gildea, R.J, Howard, J.A.K. \& Puschmann, H. (2009), J. Appl. Cryst. 42, 339-341.

2. Sheldrick, G.M. (2015). Acta Cryst. A71, 3-8.

3. Sheldrick, G.M. (2008). Acta Cryst. A64, 112-122.

\section{Crystal structure determination of Compound 16}

Crystal Data for $\mathrm{C}_{33} \mathrm{HN}_{2} \mathrm{O}_{6} \mathrm{Cl}(M=555.80 \mathrm{~g} / \mathrm{mol})$ : monoclinic, space group $\mathrm{C} 2 / \mathrm{c}$ (no. 15), $a=$ 25.162(5) $\AA, b=15.406(3) \AA, c=16.174(4) \AA, \beta=109.269(3)^{\circ}, V=5919(2) \AA^{3}, Z=8, T=296.15 \mathrm{~K}$, $\mu(\operatorname{MoK} \alpha)=0.175 \mathrm{~mm}^{-1}$, Dcalc $=1.247 \mathrm{~g} / \mathrm{cm}^{3}, 38353$ reflections measured $\left(3.15^{\circ} \leq 2 \Theta \leq 55.26^{\circ}\right), 6868$ unique $\left(R_{\text {int }}=0.0202, \mathrm{R}_{\text {sigma }}=0.0133\right)$ which were used in all calculations. The final $R_{1}$ was 0.0350 (I > $2 \sigma(\mathrm{I}))$ and $w R_{2}$ was 0.0953 (all data).

\section{Refinement model description}

Details:

Number of restraints - 0 , number of constraints - unknown.

1. Fixed Uiso

At 1.2 times of:

All $\mathrm{C}(\mathrm{H})$ groups, All $\mathrm{C}(\mathrm{H}, \mathrm{H})$ groups, All $\mathrm{N}(\mathrm{H})$ groups

At 1.5 times of:

All $\mathrm{C}(\mathrm{H}, \mathrm{H}, \mathrm{H})$ groups

2.a Ternary $\mathrm{CH}$ refined with riding coordinates:

$\mathrm{C} 4(\mathrm{H} 4), \mathrm{C} 3(\mathrm{H} 3), \mathrm{C} 27(\mathrm{H} 27), \mathrm{C} 31(\mathrm{H} 31)$

2 .b Secondary $\mathrm{CH} 2$ refined with riding coordinates: C19 (H19A, H19B)

2.C Aromatic/amide $\mathrm{H}$ refined with riding coordinates:

$\mathrm{N} 2(\mathrm{H} 2), \mathrm{N} 1(\mathrm{H} 1), \mathrm{C} 6(\mathrm{H} 6), \mathrm{C} 9(\mathrm{H} 9), \mathrm{C} 10(\mathrm{H} 10), \mathrm{C} 13(\mathrm{H} 13), \mathrm{C} 16(\mathrm{H} 16), \mathrm{C} 17(\mathrm{H} 17)$,

$\mathrm{C} 11(\mathrm{H} 11), \mathrm{C} 21(\mathrm{H} 21), \mathrm{C} 24(\mathrm{H} 24), \mathrm{C} 25(\mathrm{H} 25), \mathrm{C} 22(\mathrm{H} 22)$

2.d Idealised Me refined as rotating group:

C26 (H26A, H26B, H26C) , С 33 (H33A, H33B, H33C), С28(H28A, H28B, H28C), С 32 (H32A, H32B, $\mathrm{H} 32 \mathrm{C})$, C30(H30A, H30B, H30C), C29(H29A, H29B, H29C) 


\section{HPLC Traces}

Synthetic Bisavenanthramide B-6

\section{$====$ Shimadzu LCsolution Analysis Report ====}

Acquired by
Sample Name
Sample 10
Vail \#
Injection Volume
Data File Name
Method File Name
Batch File Name
Report File Name
Data Acquired
Data Processed

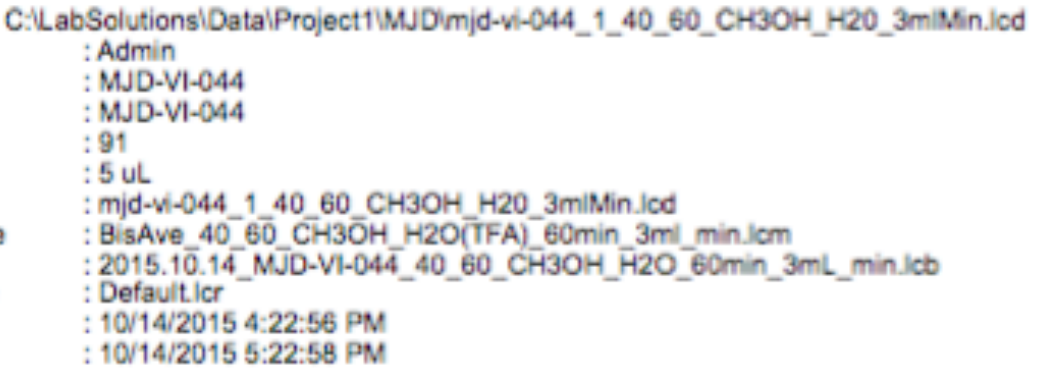

\section{$<$ Chromatogram $>$}

$m V$

C:LabSolutionsiDatalProject1M.JDimjd-vi-044_1_40_60_CH3OH_H20_3miMin.lod

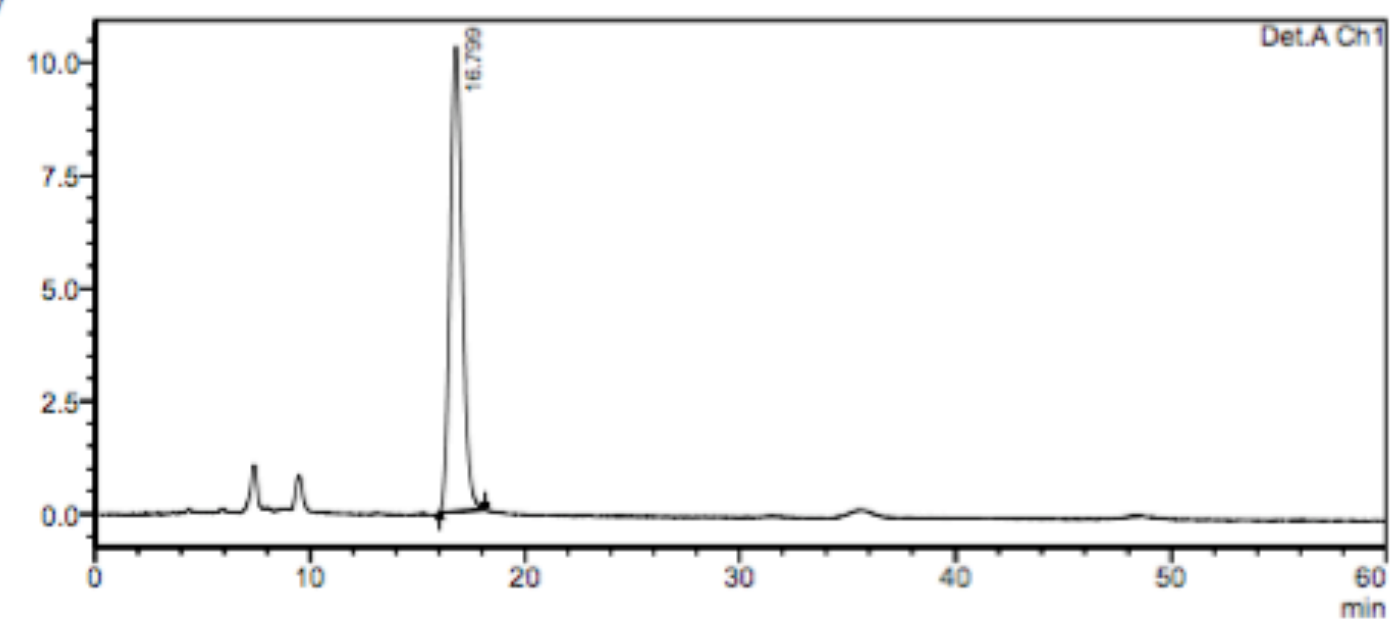

$\mathrm{mV}$

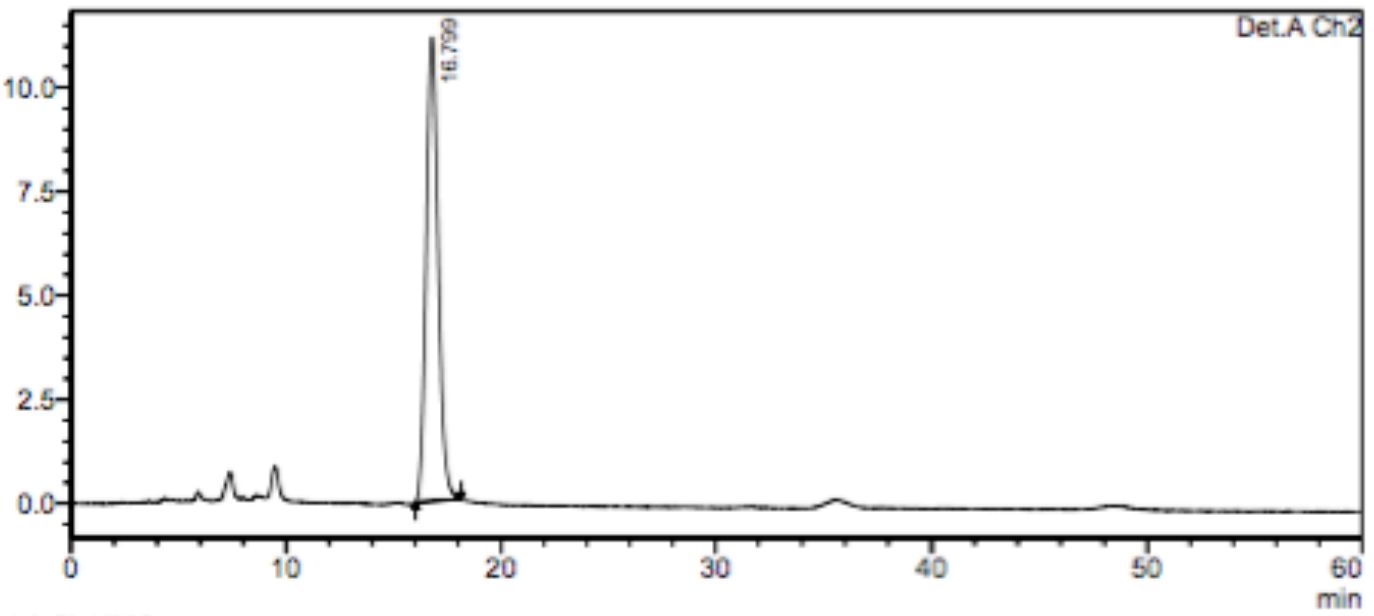

1 Det.A Ch $1 / 308 \mathrm{~nm}$

2 Det.A Ch2/254nm 
Natural Bisavenanthramide B-6

\section{==== Shimadzu LCsolution Analysis Report ====}

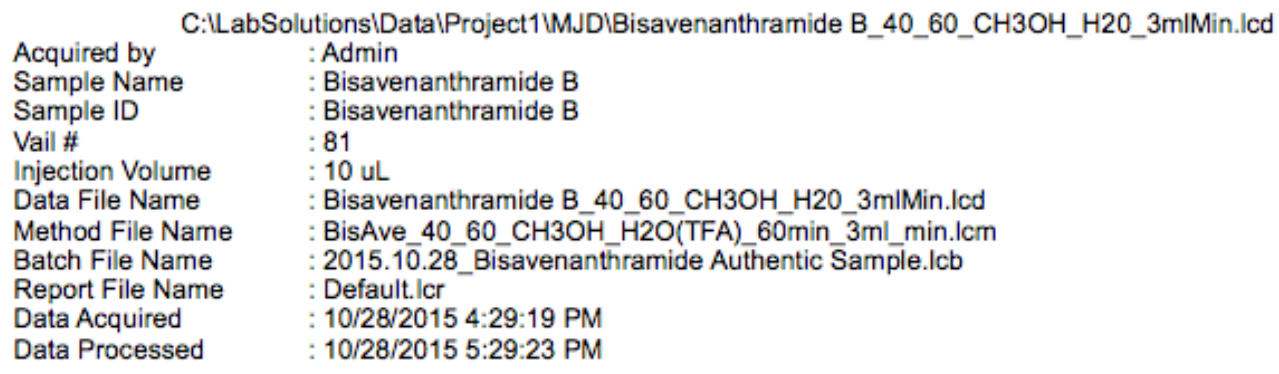

$<$ Chromatogram>

$\mathrm{mV}$

C:ILabSolutionsiDatalProject1MJDiBisavenanthramide B_40_60_CH3OH_H20_3mIMin.lcd

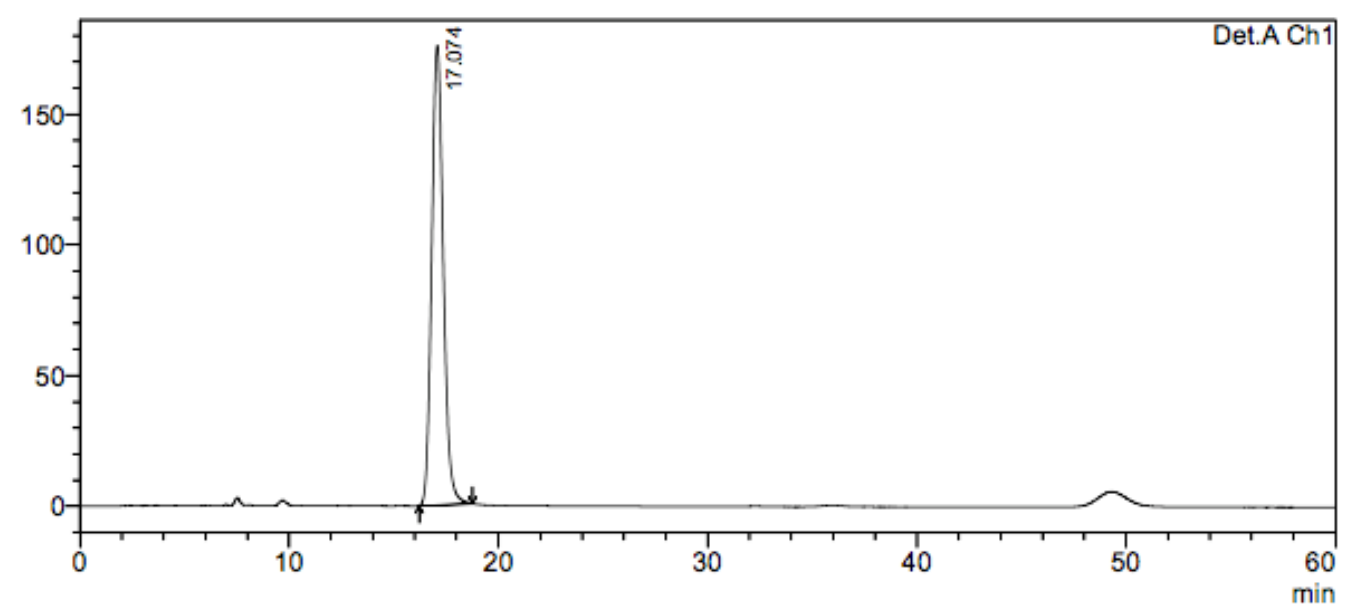

$\mathrm{mV}$

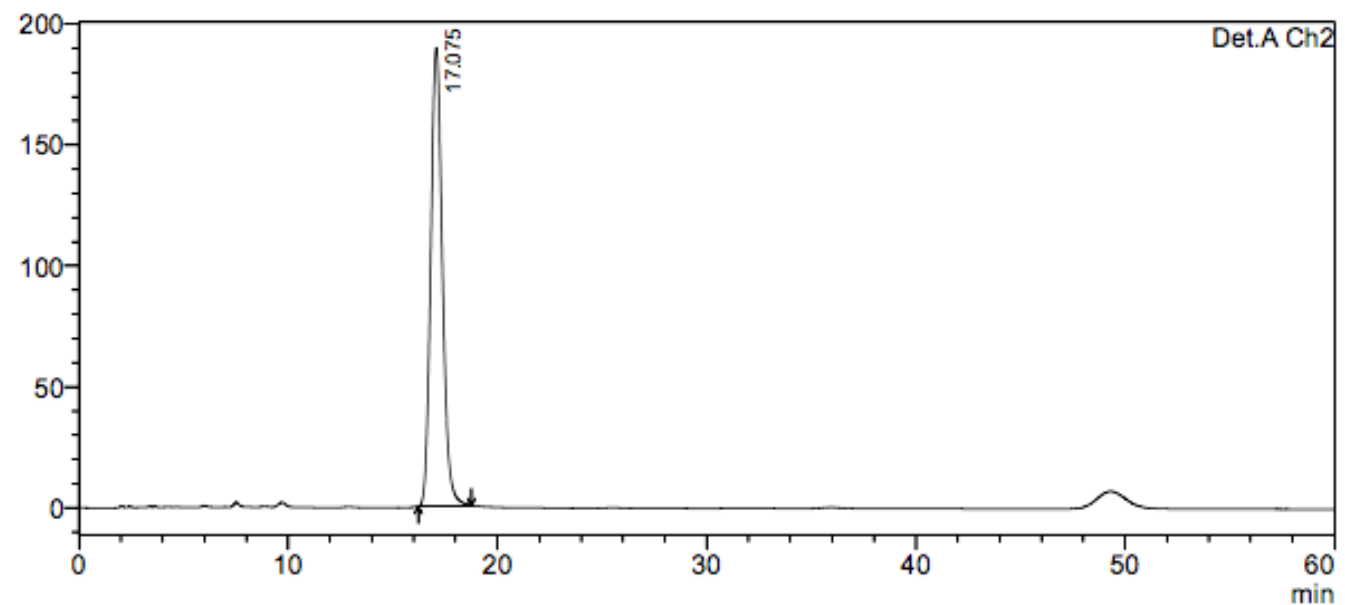

1 Det.A Ch $1 / 308$ nm

2 Det.A Ch2/254nm 
Coinjection of synthetic and natural material

\section{==== Shimadzu LCsolution Analysis Report ====}

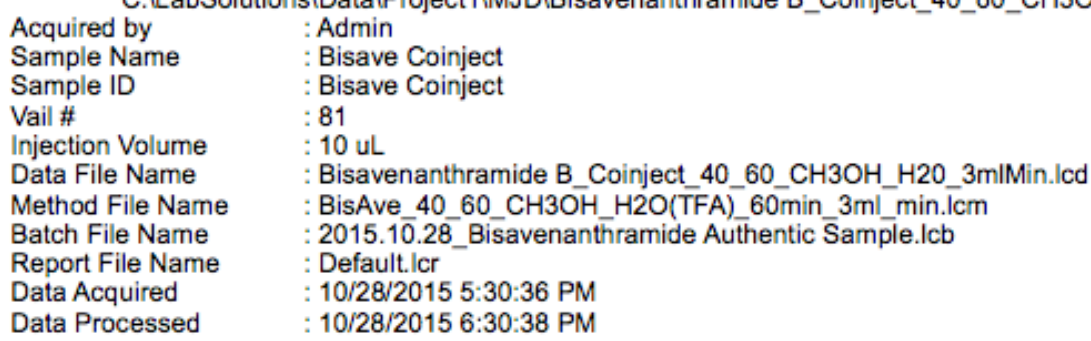

$<$ Chromatogram>

$\mathrm{mV}$

C:LabSolutionsiDatalProject11MJDIBisavenanthramide B_Coinject_40_60_CH3OH_H20_3mIMin.lcd

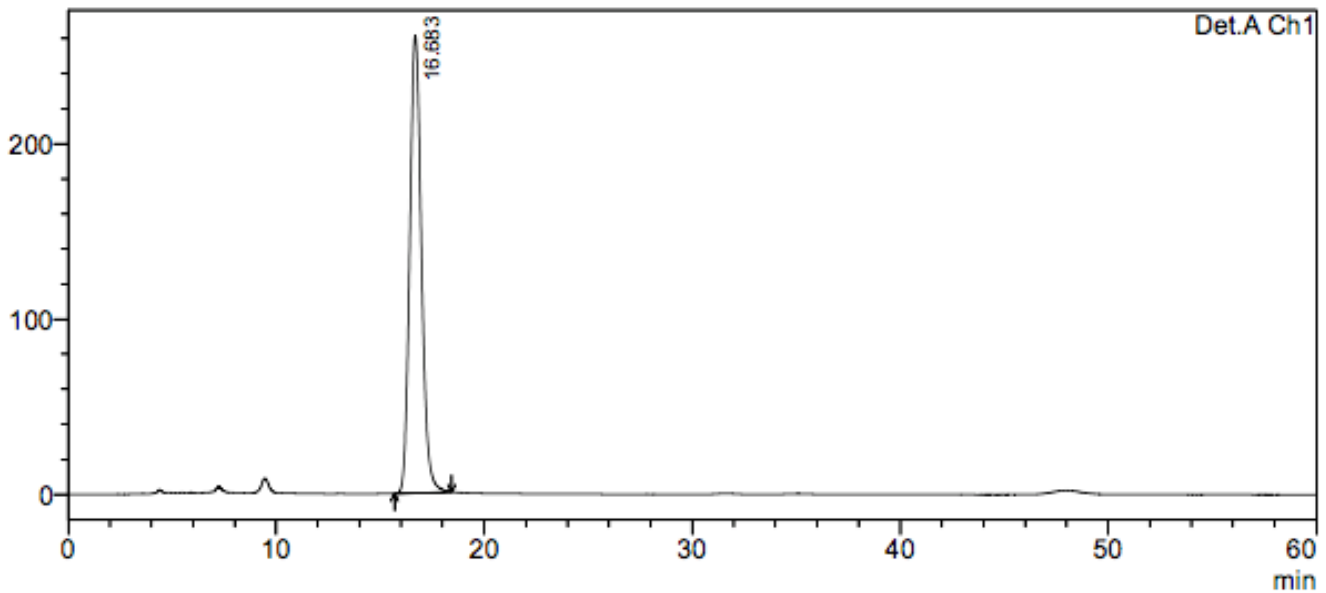

$\mathrm{mV}$

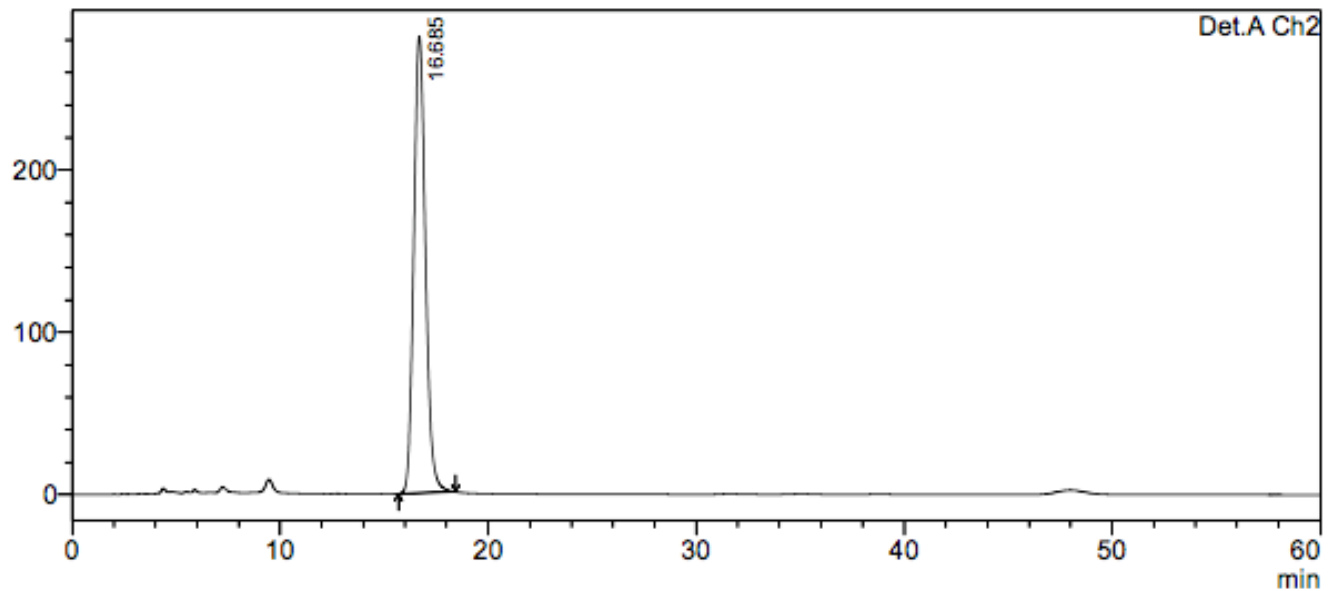

1 Det.A Ch $1 / 308 \mathrm{~nm}$

2 Det.A Ch2/254nm 


\section{Spectral Comparisons}

\begin{tabular}{|c|c|c|c|}
\hline $\begin{array}{l}\text { 1H Reported } \\
\text { (300 MHz) }\end{array}$ & $\begin{array}{l}{ }^{1} \mathrm{H} \text { Synthetic } \\
(600 \mathrm{MHz})\end{array}$ & $\begin{array}{c}{ }^{13} \mathrm{C} \text { Reported } \\
(75 \mathrm{MHz})\end{array}$ & $\begin{array}{c}{ }^{13} \mathrm{C} \text { Synthetic } \\
(151 \mathrm{MHz})\end{array}$ \\
\hline $3.73(3 \mathrm{H}, \mathrm{s})$ & $3.73(\mathrm{~s}, 3 \mathrm{H})$ & $170.7(2)$ & $170.7(2)$ \\
\hline $3.76(3 \mathrm{H}, \mathrm{s})$ & $3.76(\mathrm{~s}, 3 \mathrm{H})$ & 170.1 & 170.1 \\
\hline $4.28(1 \mathrm{H}, \mathrm{m})$ & $4.28(\mathrm{dd}, J=3.8,2.4 \mathrm{~Hz}, 1 \mathrm{H})$ & 168.4 & 168.4 \\
\hline $5.33(1 \mathrm{H}, \mathrm{d}, J=3.6 \mathrm{~Hz})$ & $5.33(\mathrm{~d}, J=3.6 \mathrm{~Hz}, 1 \mathrm{H})$ & 157.9 & 157.9 \\
\hline $6.73(1 \mathrm{H}, \mathrm{d}, J=8.1 \mathrm{~Hz})$ & $6.73(\mathrm{~d}, J=8.1 \mathrm{~Hz}, 1 \mathrm{H})$ & 154.6 & 154.5 \\
\hline $6.75-6.90(4 \mathrm{H}, \mathrm{m})$ & $6.76^{-6.88}(\mathrm{~m}, 4 \mathrm{H})$ & 149.7 & 149.7 \\
\hline $6.94(1 \mathrm{H}, \mathrm{dd}, J=9.0,3.0 \mathrm{~Hz})$ & $6.95(\mathrm{dd}, J=9.0,3.0 \mathrm{~Hz}, 1 \mathrm{H})$ & 149.4 & 149.4 \\
\hline $6.99(1 \mathrm{H}, \mathrm{bs})$ & $6.99(\mathrm{bs}, 1 \mathrm{H})$ & 149.1 & 149.0 \\
\hline $7.04(1 \mathrm{H}, \mathrm{dd}, J=8.3,1.7 \mathrm{~Hz})$ & $7.04(\mathrm{dd}, J=8.2,2.0 \mathrm{~Hz}, 1 \mathrm{H})$ & 147.9 & 147.9 \\
\hline $7.07(1 \mathrm{H}, \mathrm{d}, J=1.7 \mathrm{~Hz})$ & $7.08(\mathrm{~d}, J=1.9 \mathrm{~Hz}, 1 \mathrm{H})$ & 138.2 & 138.2 \\
\hline $7.36(1 \mathrm{H}, \mathrm{d}, J=2.7 \mathrm{~Hz})$ & $7.37(\mathrm{~d}, J=2.6 \mathrm{~Hz}, 1 \mathrm{H})$ & 133.6 & 133.6 \\
\hline $7.38(1 \mathrm{H}, \mathrm{d}, J=3.0 \mathrm{~Hz})$ & $7.38(\mathrm{~d}, J=3.0 \mathrm{~Hz}, 1 \mathrm{H})$ & 132.8 & 132.8 \\
\hline $7.69(1 \mathrm{H}, \mathrm{d}, J=2.2 \mathrm{~Hz})$ & $7.70(\mathrm{~d}, J=2.4 \mathrm{~Hz}, 1 \mathrm{H})$ & $131.1(2)$ & $131.1(2)$ \\
\hline \multirow[t]{17}{*}{$8.23(1 \mathrm{H}, \mathrm{d}, J=9.0 \mathrm{~Hz})$} & $8.24(\mathrm{~d}, J=9.0 \mathrm{~Hz}, 1 \mathrm{H})$ & 129.5 & 129.5 \\
\hline & & 127.6 & 127.6 \\
\hline & & 126.3 & 126.3 \\
\hline & & 125.9 & 125.9 \\
\hline & & 123.8 & 123.8 \\
\hline & & 121.7 & 121.7 \\
\hline & & 121.6 & 121.6 \\
\hline & & 120.4 & 120.4 \\
\hline & & 120.1 & 120.1 \\
\hline & & 118.8 & 118.8 \\
\hline & & 118.1 & 118.0 \\
\hline & $\mathrm{OH}$ & 116.5 & 116.5 \\
\hline & & 116.3 & 116.3 \\
\hline & & 113.5 & 113.5 \\
\hline & & 111.8 & 111.8 \\
\hline & & 69.2 & 69.2 \\
\hline & & 57.1 & 57.1 \\
\hline \multirow{2}{*}{\multicolumn{2}{|c|}{ Bisavenanthramide B-6 }} & 56.4 & 56.4 \\
\hline & & 56.3 & 56.3 \\
\hline
\end{tabular}


${ }^{1} \mathrm{H} \mathrm{NMR}\left(600 \mathrm{MHz}, \mathrm{CDCl}_{3}\right.$ )
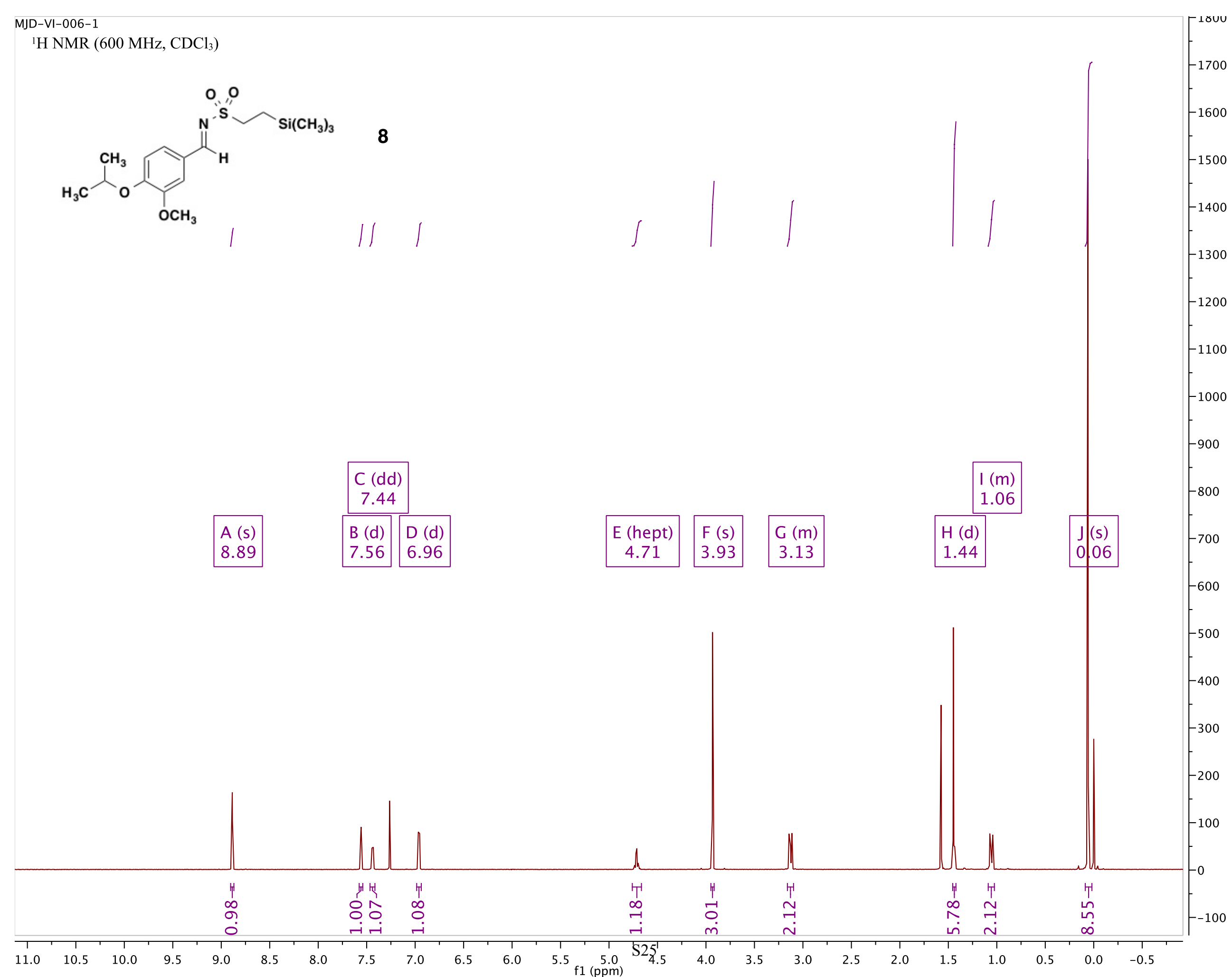

- 1000 
${ }^{13} \mathrm{C}$ NMR (151 MHz, $\left.\mathrm{CDCl}_{3}\right)$<smiles>COc1cc(/C=N/S(=O)(=O)CC[Si])ccc1OC(C)C</smiles> 
${ }^{1} \mathrm{H}$ NMR (600 MHz, DMSO)<smiles>COc1cc(/C=C(\CC(=O)O)C(=O)O)ccc1OC(C)C</smiles>

$9 a$

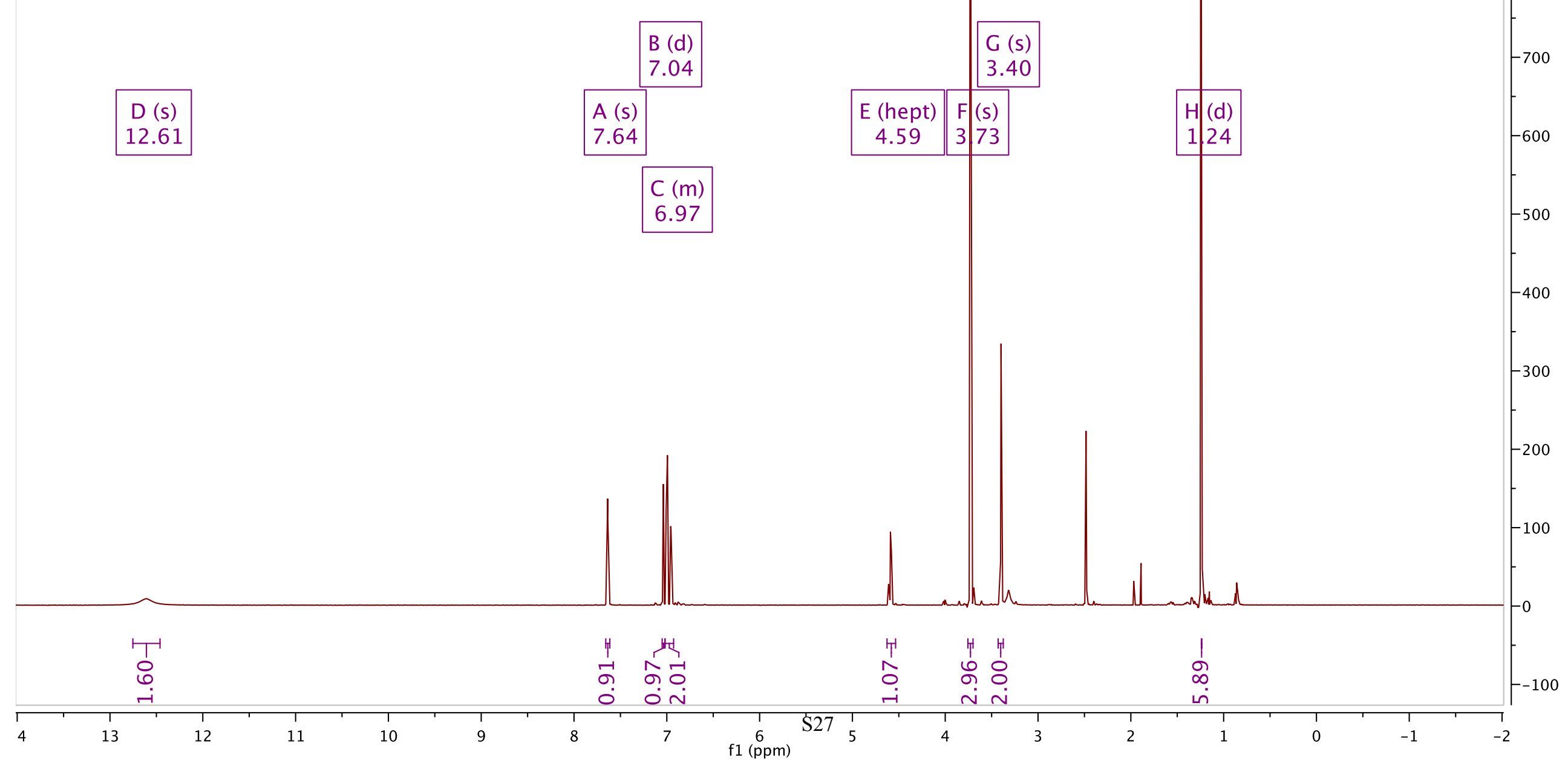


${ }^{13} \mathrm{C}$ NMR (151 MHz, DMSO)<smiles>COc1cc(/C=C(\CC(=O)O)C(=O)O)ccc1OC(C)C</smiles> 


\section{${ }^{1} \mathrm{H}$ NMR $\left(600 \mathrm{MHz}, \mathrm{CDCl}_{3}\right)$}
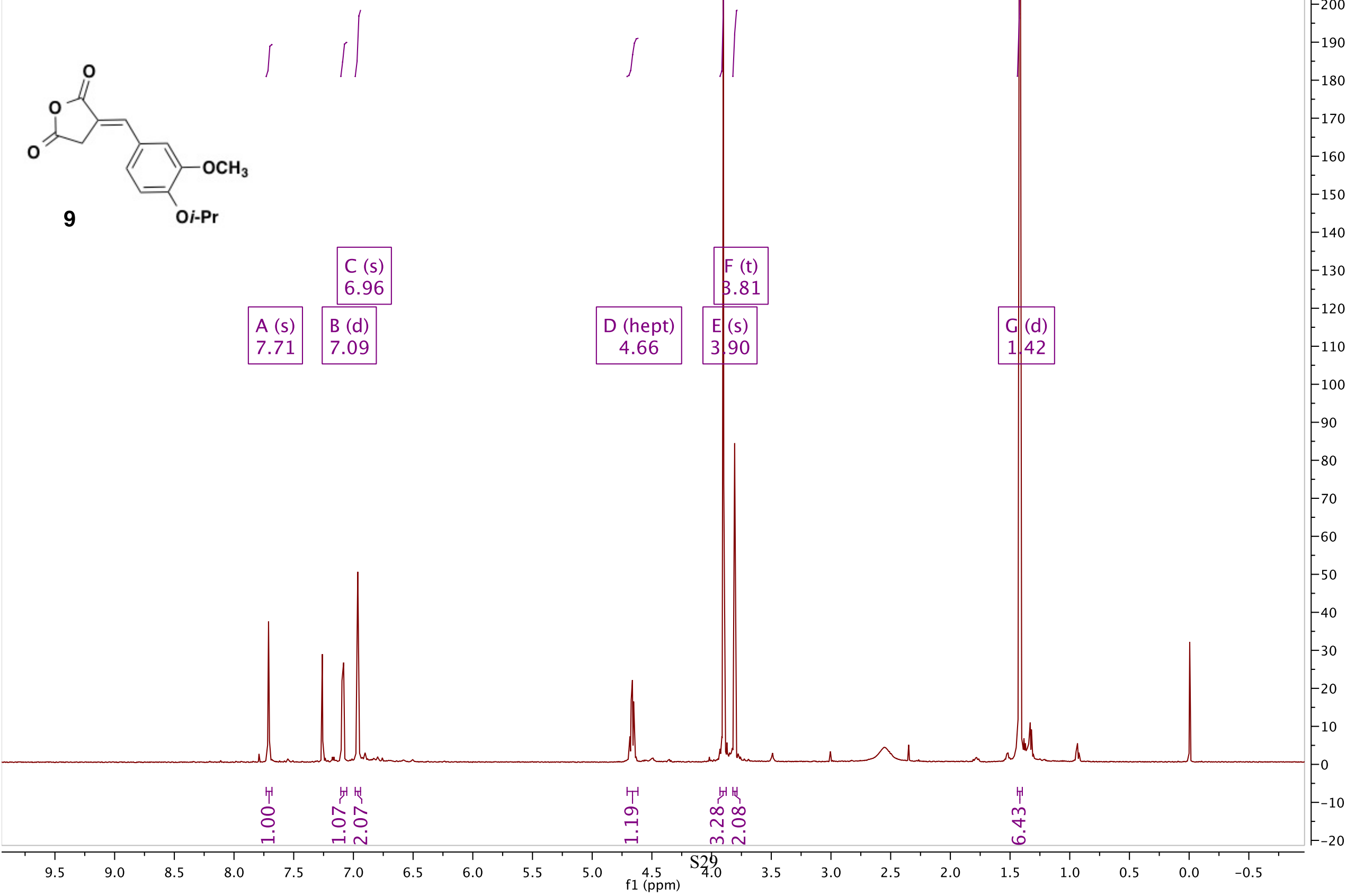
${ }^{13} \mathrm{C}$ NMR (151 MHz, $\left.\mathrm{CDCl}_{3}\right)$<smiles>CCCOc1ccc(/C=C2\CC(=O)OC2=O)cc1OC</smiles> 
${ }^{1} \mathrm{H} \mathrm{NMR}\left(600 \mathrm{MHz}, \mathrm{CDCl}_{3}\right.$ )

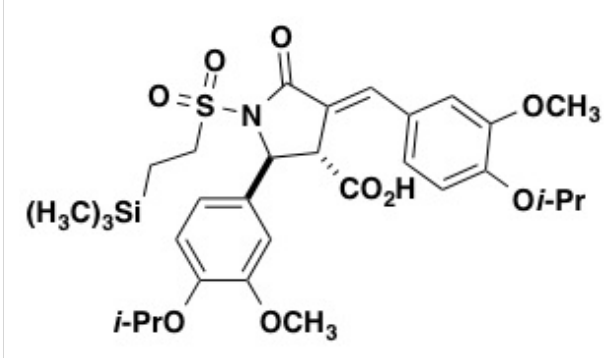

11
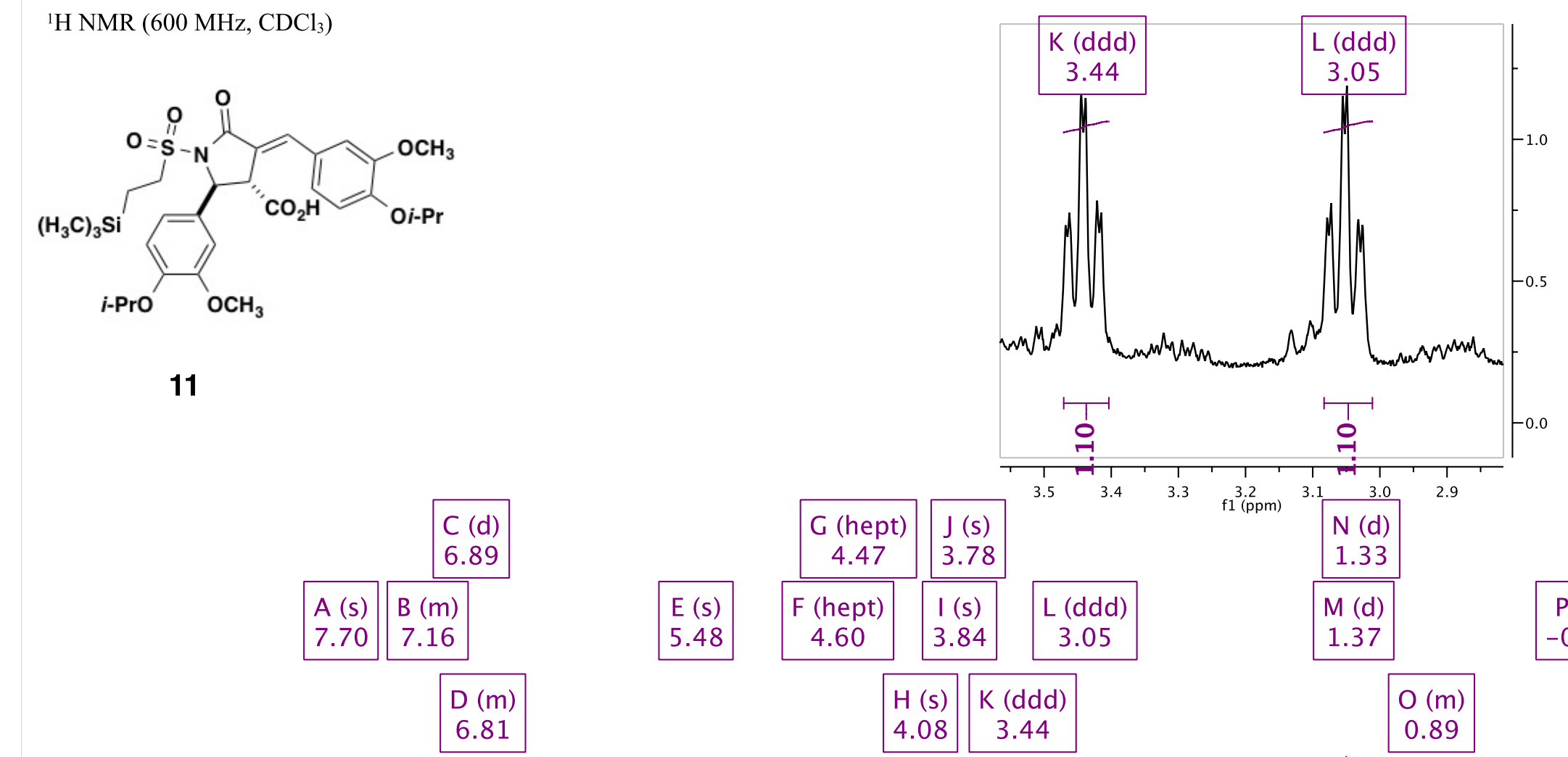

$3.5 \quad 3.4$
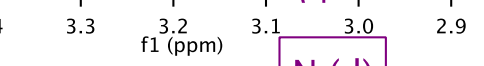

\begin{tabular}{|c|c|c|}
\hline \begin{tabular}{l|l|} 
F (hept) \\
4.60
\end{tabular} & $\begin{array}{c}\text { I (s) } \\
3.84\end{array}$ & $\begin{array}{c}L \text { (ddd) } \\
3.05\end{array}$ \\
\hline
\end{tabular}

H (s) K (ddd)

$4.08 \quad 3.44$

3.05
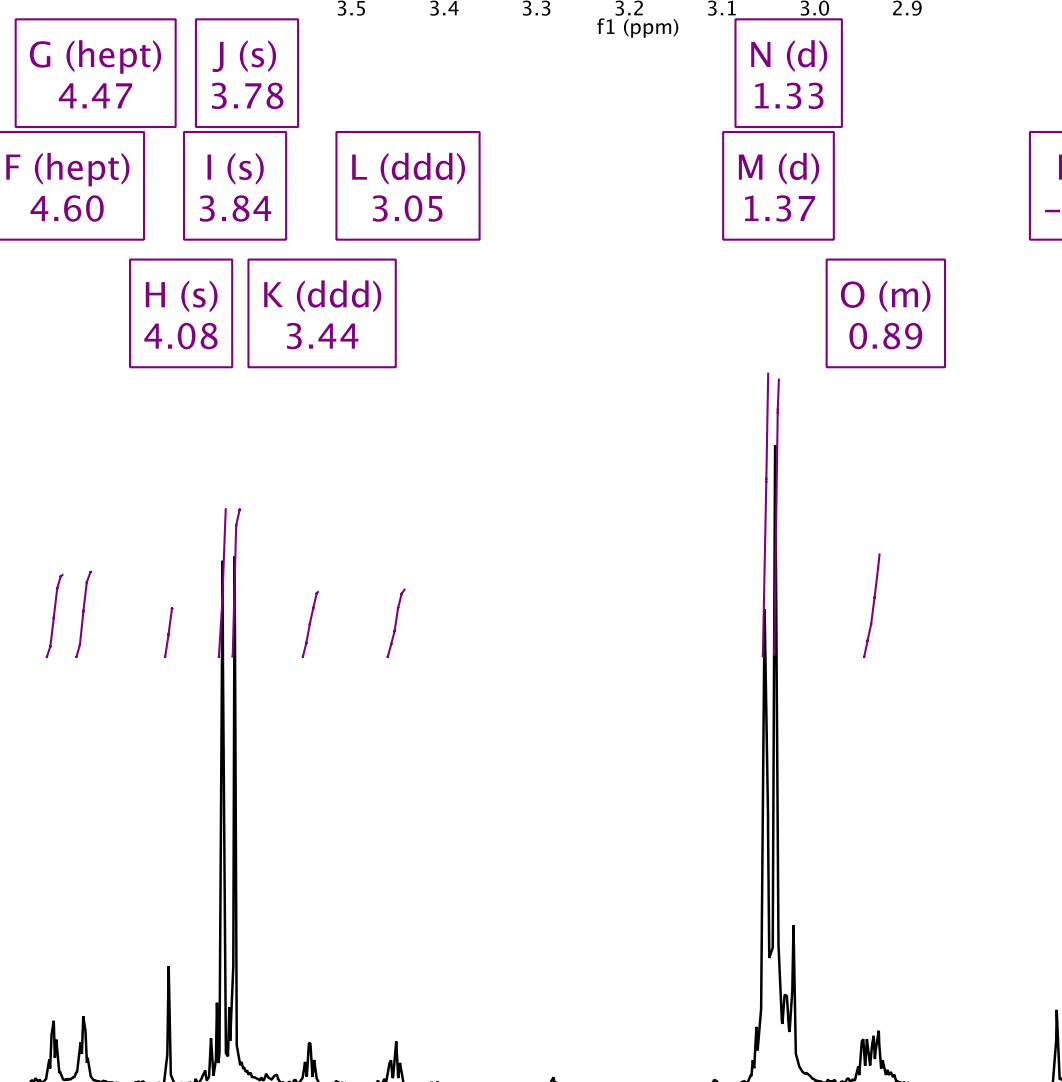

11

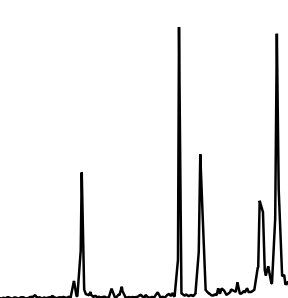

H $\quad r n$

군

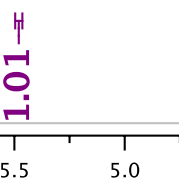

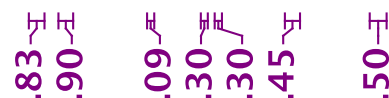

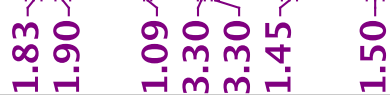

${ }_{4.5}, \mathrm{~S}_{3.5}$ 


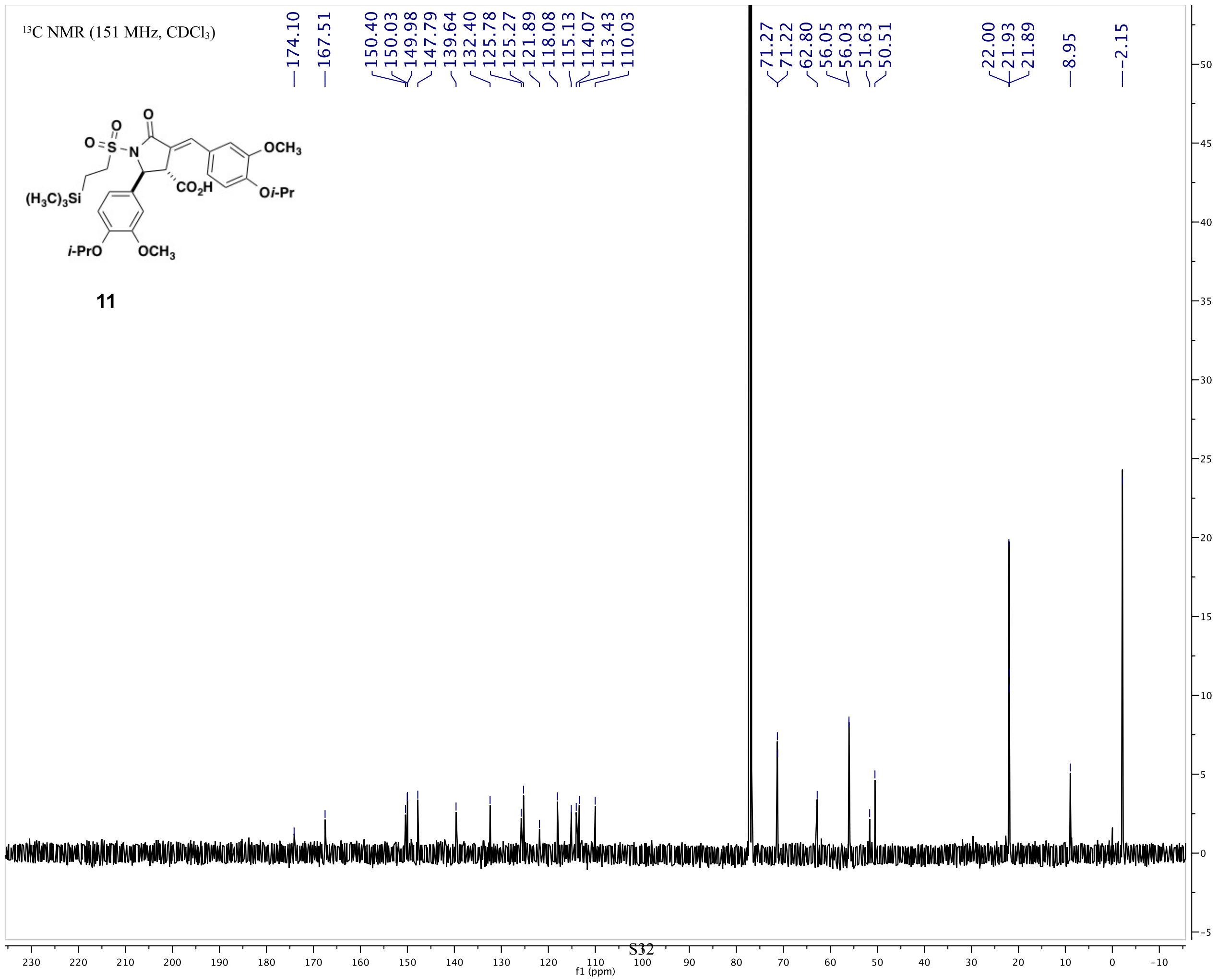


${ }^{1} \mathrm{H} \mathrm{NMR}\left(600 \mathrm{MHz}, \mathrm{CDCl}_{3}\right.$ )
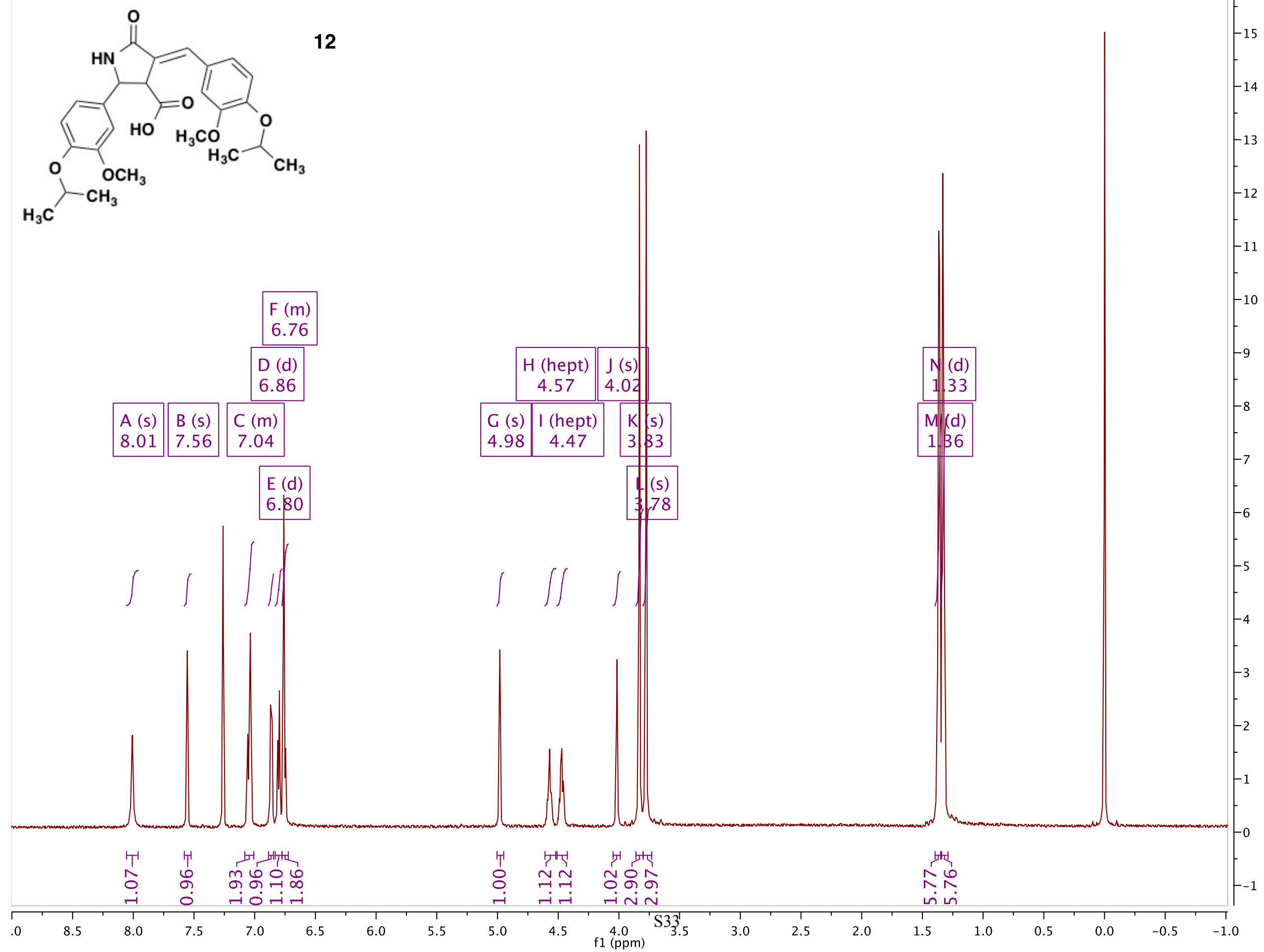
${ }^{13} \mathrm{C}$ NMR $\left(151 \mathrm{MHz}, \mathrm{CDCl}_{3}\right)$

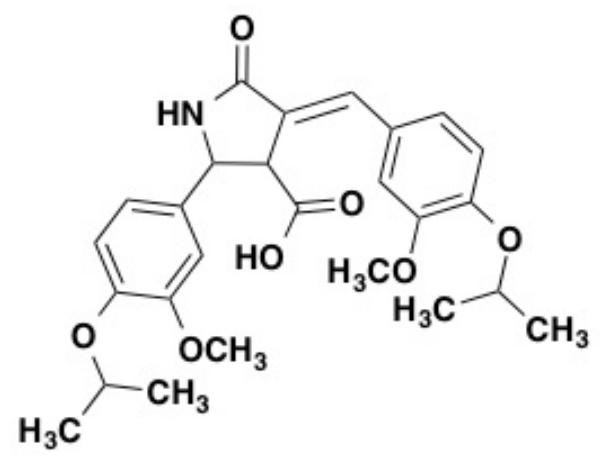

12

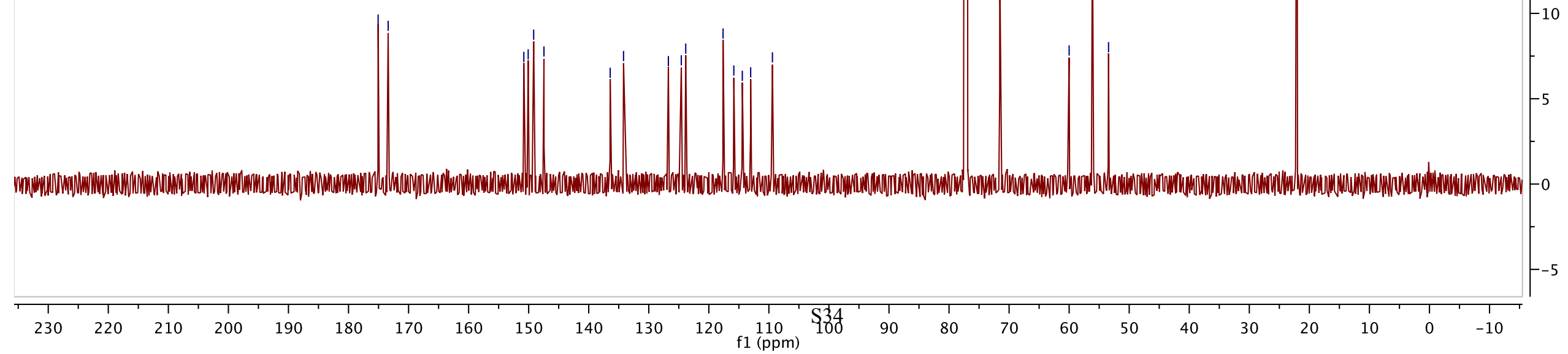




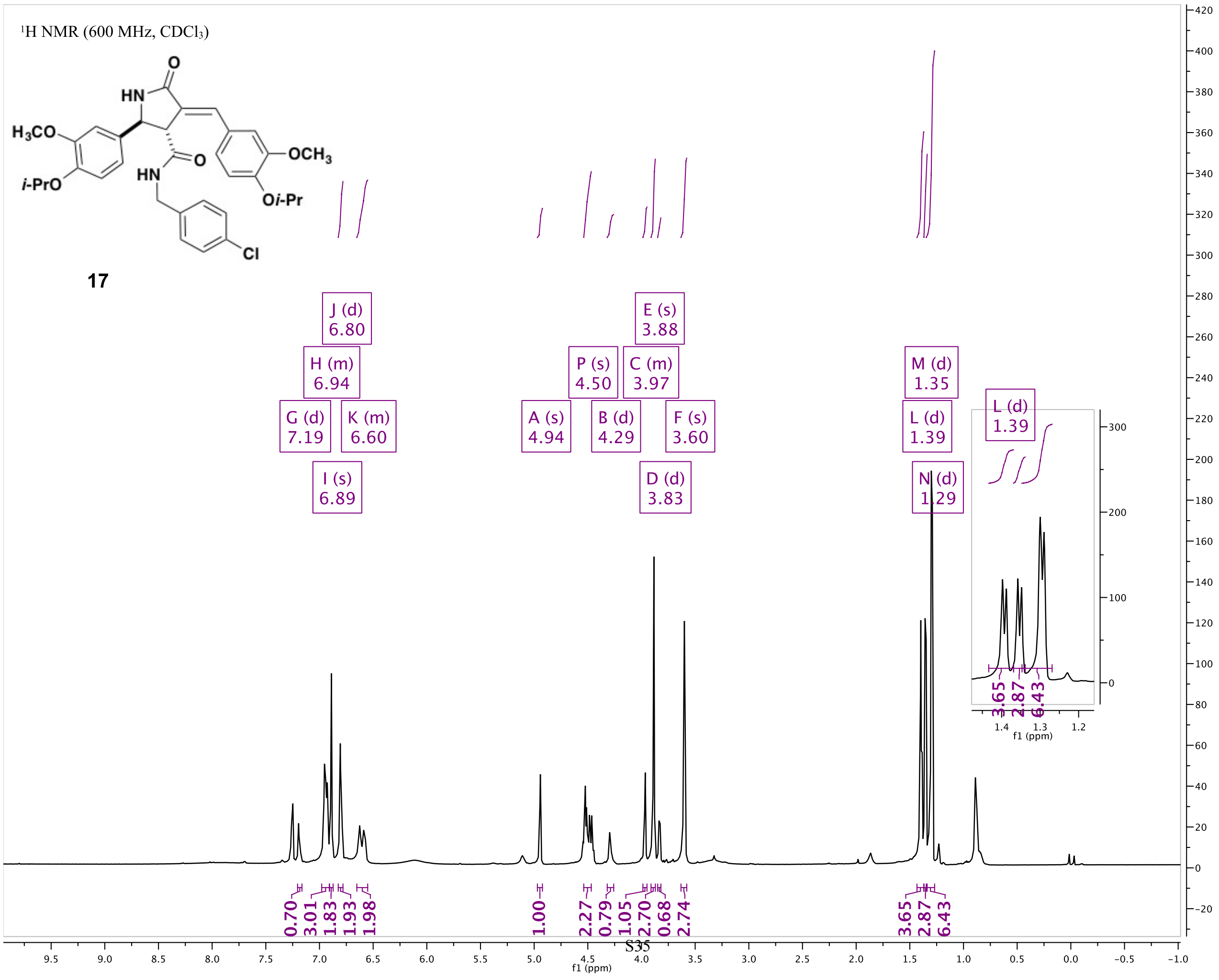


${ }^{13} \mathrm{C}$ NMR (151 MHz, $\left.\mathrm{CDCl}_{3}\right)$

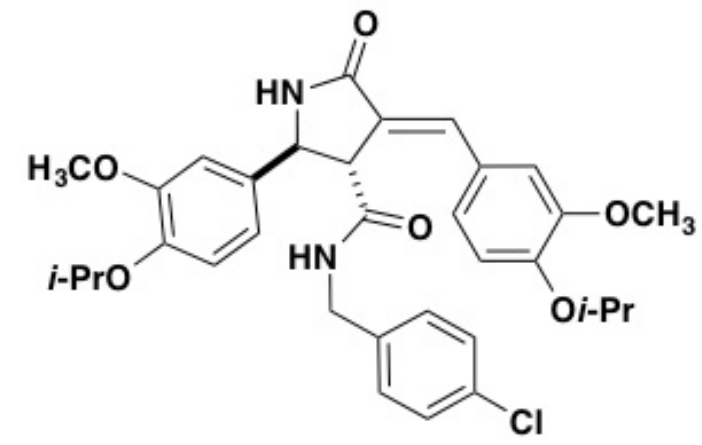

17

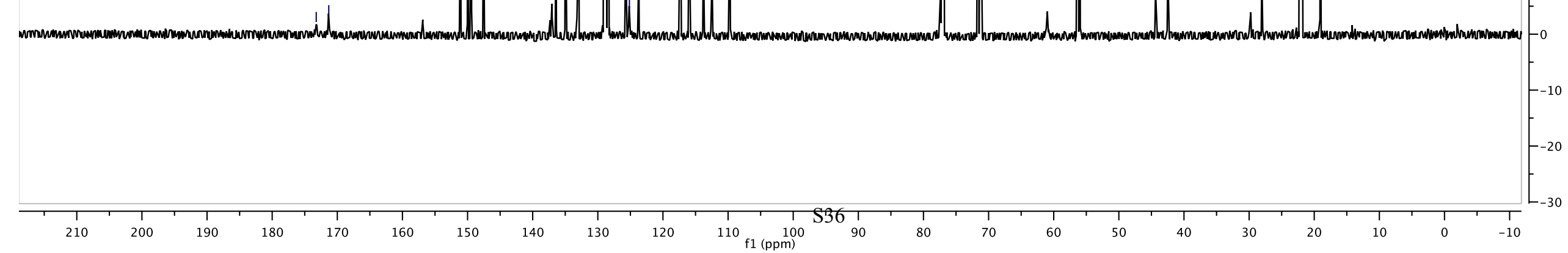


${ }^{1} \mathrm{H}$ NMR (600 MHz, $\mathrm{CDCl}_{3}$ )

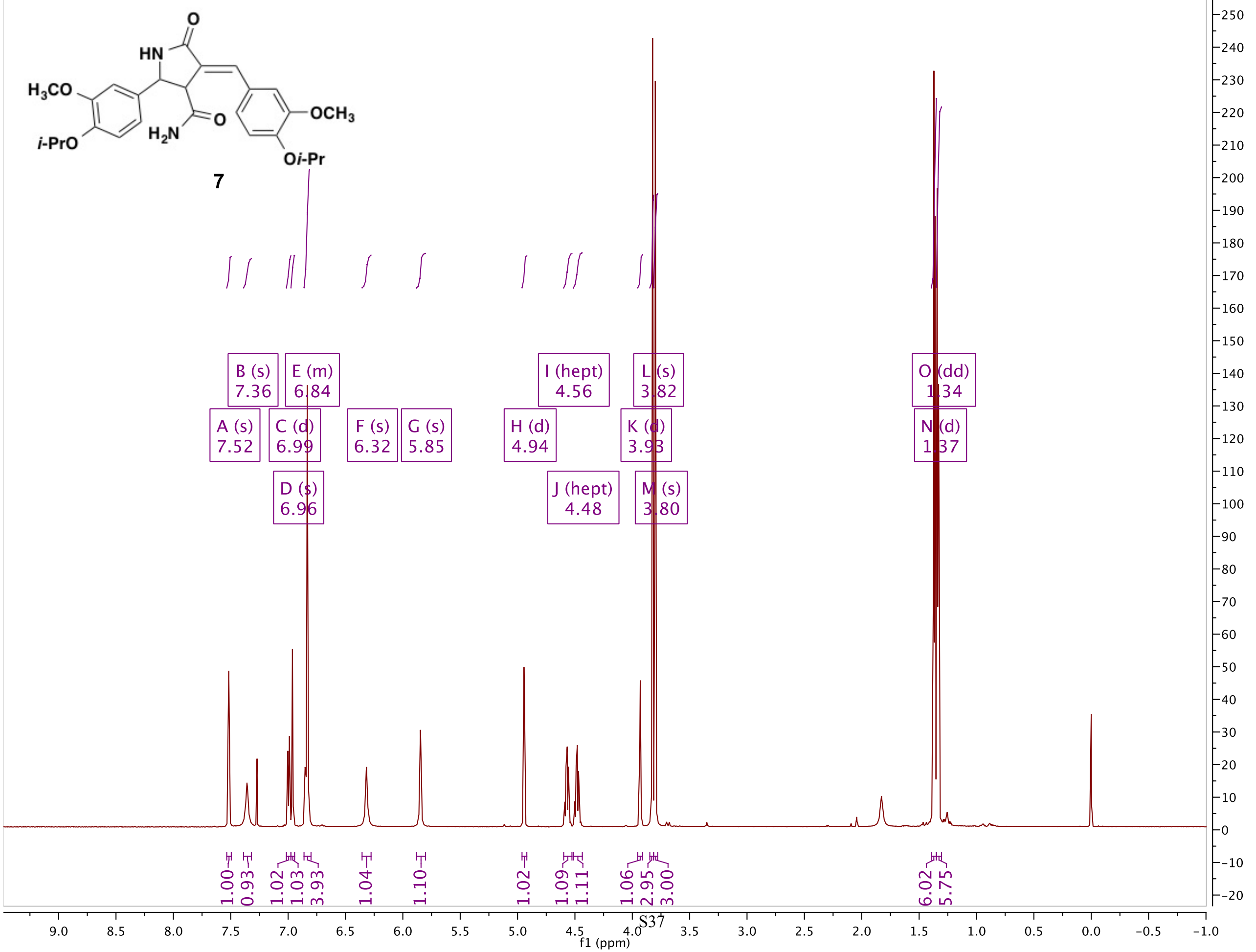


<smiles>CCCOc1ccc(/C=C2/C(=O)NC(c3ccc(OC(C)C)c(OC)c3)C2C(N)=O)cc1OC</smiles>

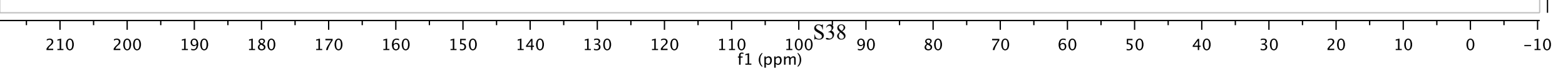


${ }^{1} \mathrm{H}$ NMR $\left(600 \mathrm{MHz}, \mathrm{CDCl}_{3}\right)$
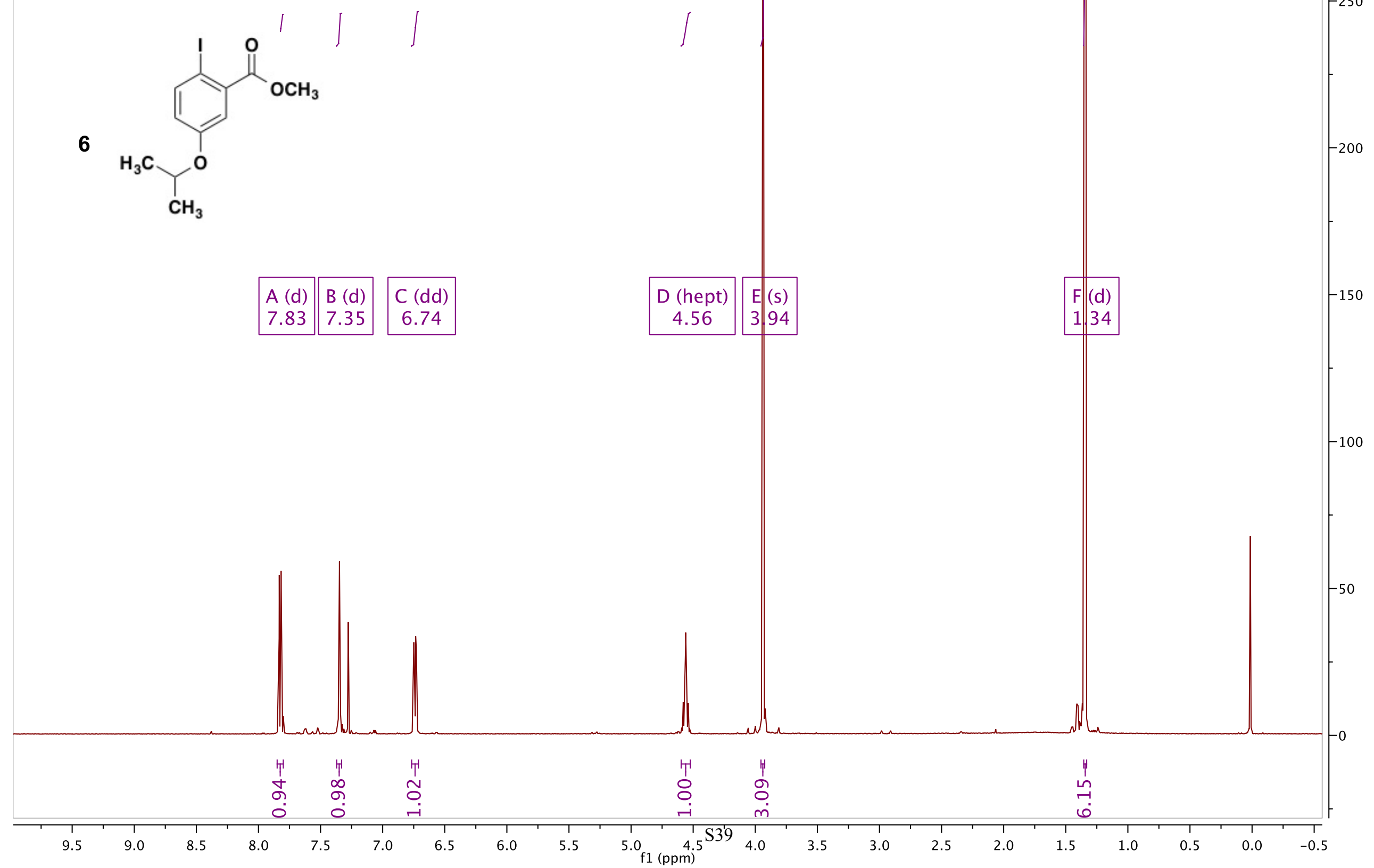
${ }^{13} \mathrm{C}$ NMR (151 MHz, $\mathrm{CDCl}_{3}$ )<smiles>COC(=O)c1cc(OC(C)C)ccc1I</smiles>

6

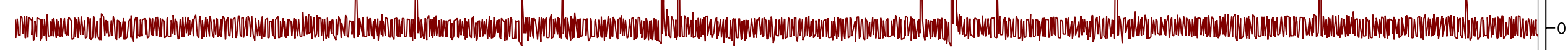

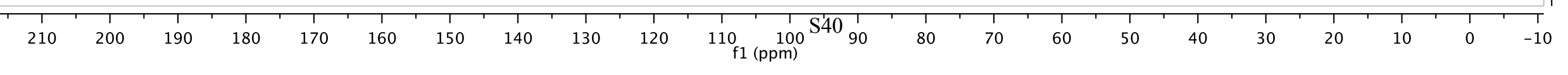


${ }^{1} \mathrm{H} \mathrm{NMR}\left(600 \mathrm{MHz}, \mathrm{CDCl}_{3}\right)$

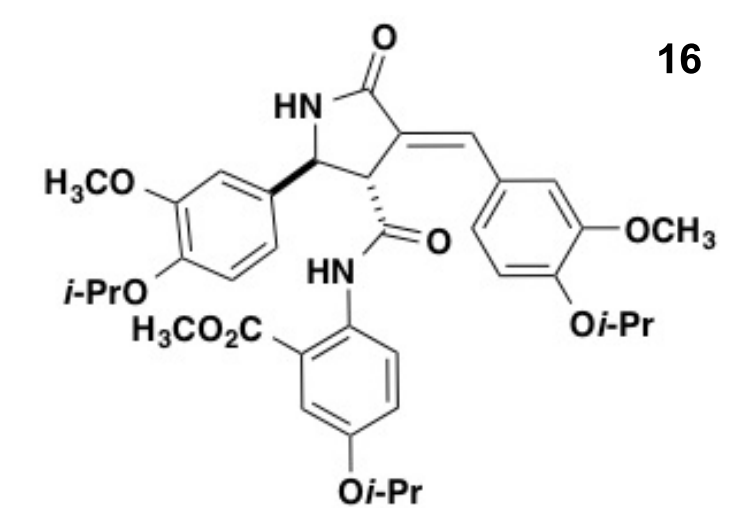

16
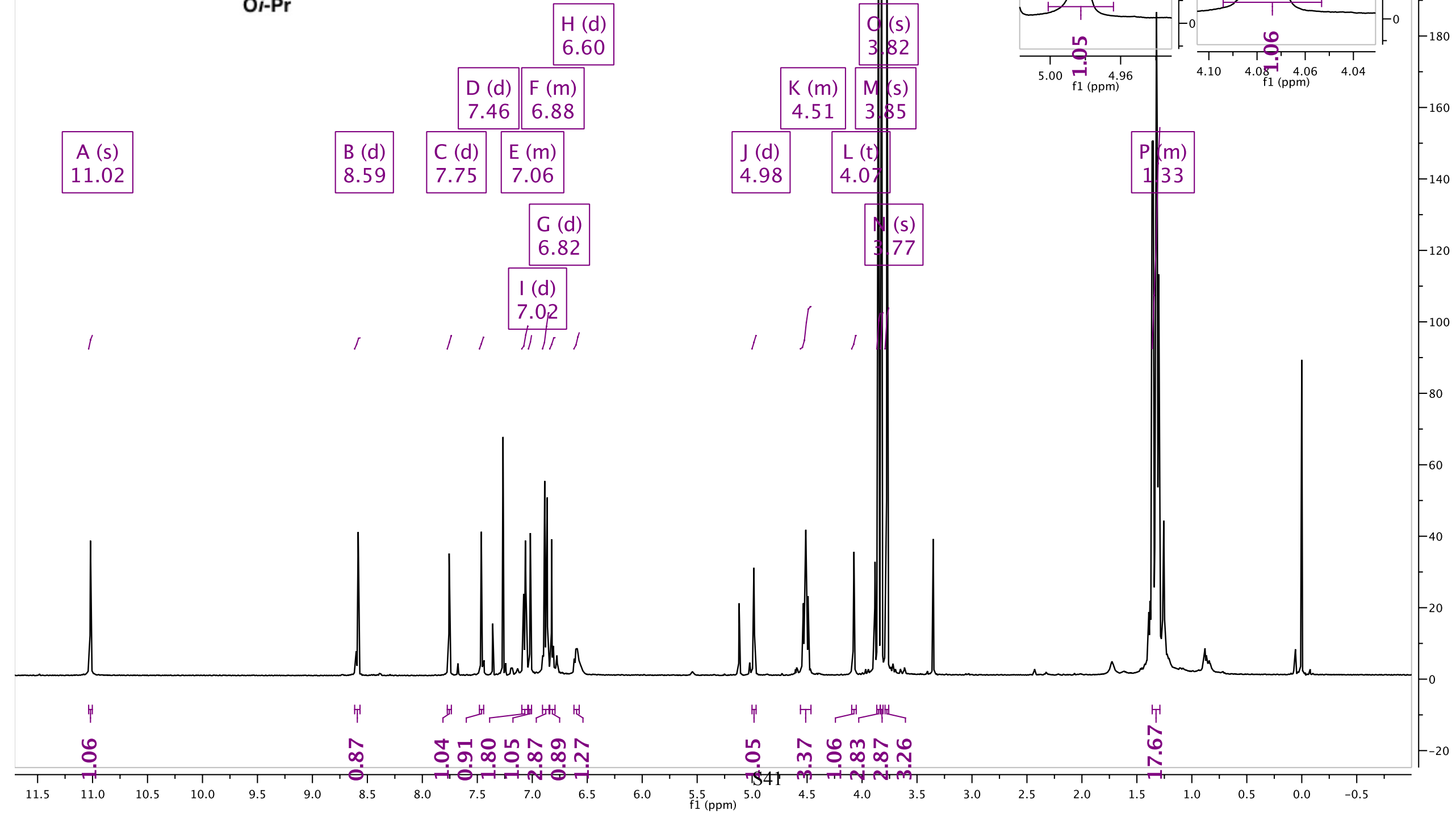

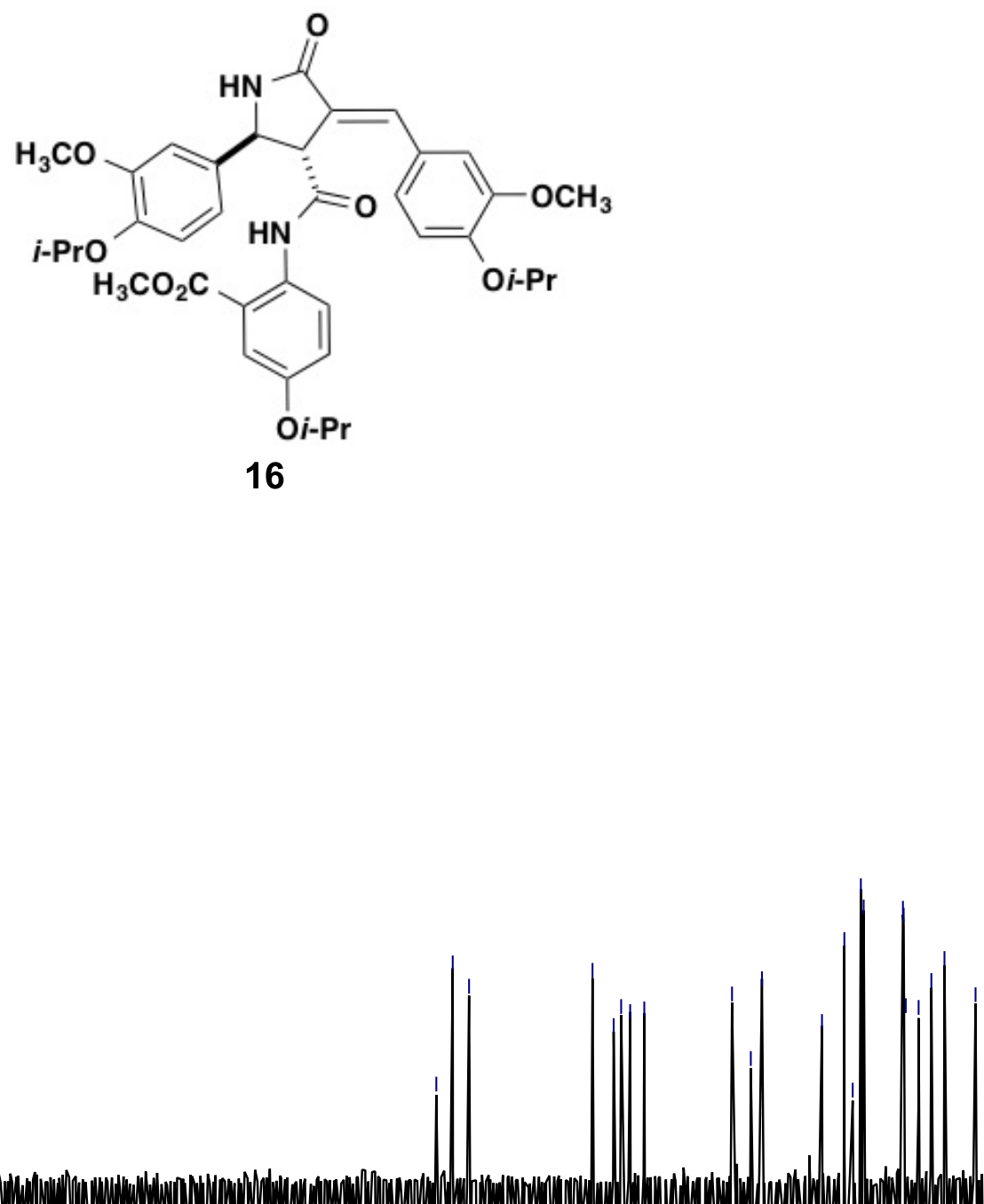


\section{${ }^{1} \mathrm{H}$ NMR (800 MHz. $\mathrm{CDCl}_{2}$ )}<smiles>CCCOc1ccc(NC(=O)[C@@H]2/C(=C\c3ccc(OCCC)c(OC)c3)C(=O)N(c3ccc(OCCC)cc3C(=O)OC)[C@@H]2c2ccc(OCCC)c(OC)c2)c(C(=O)OC)c1</smiles>

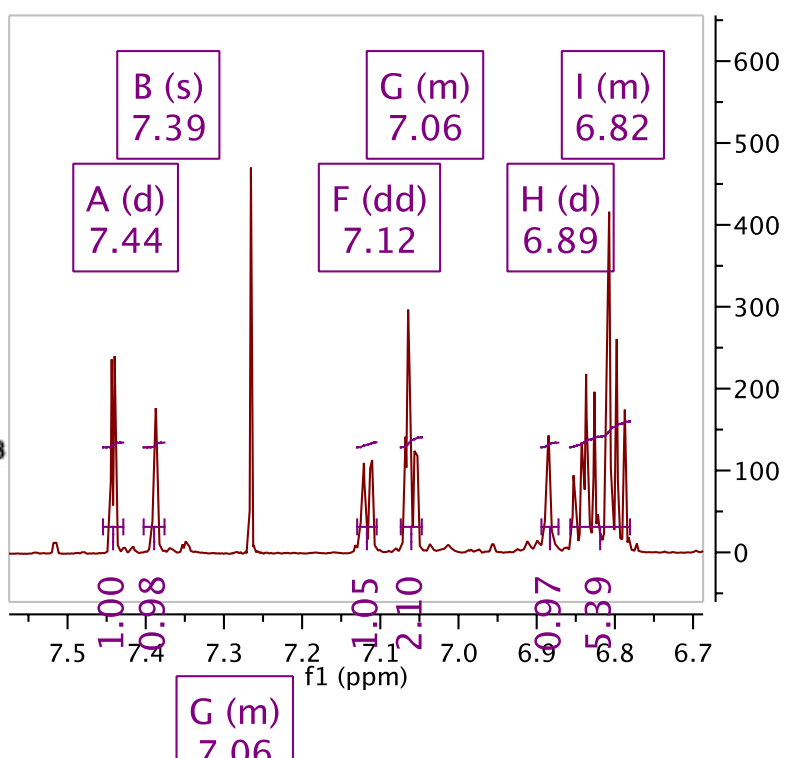



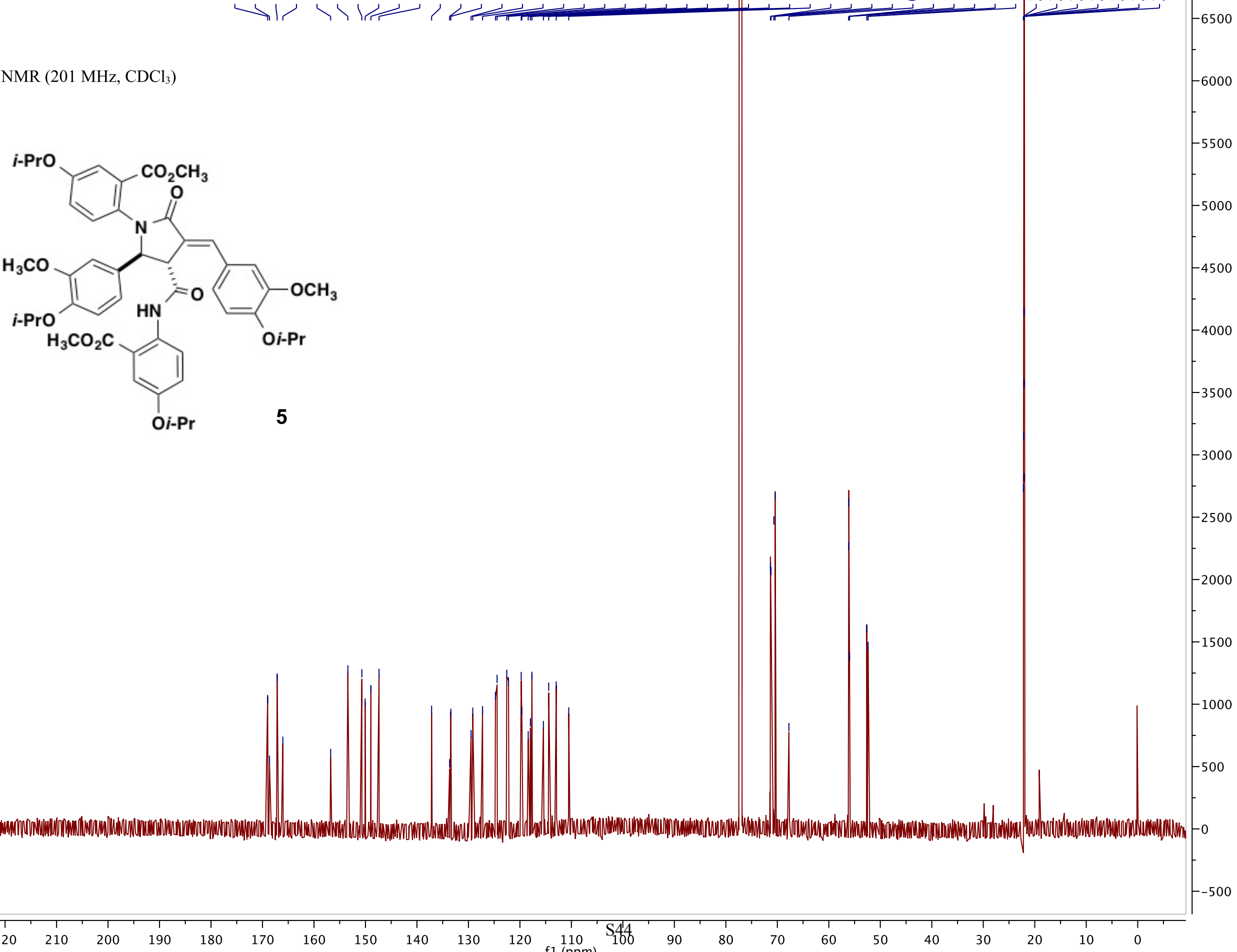

220 
MJD-VI-074c-3

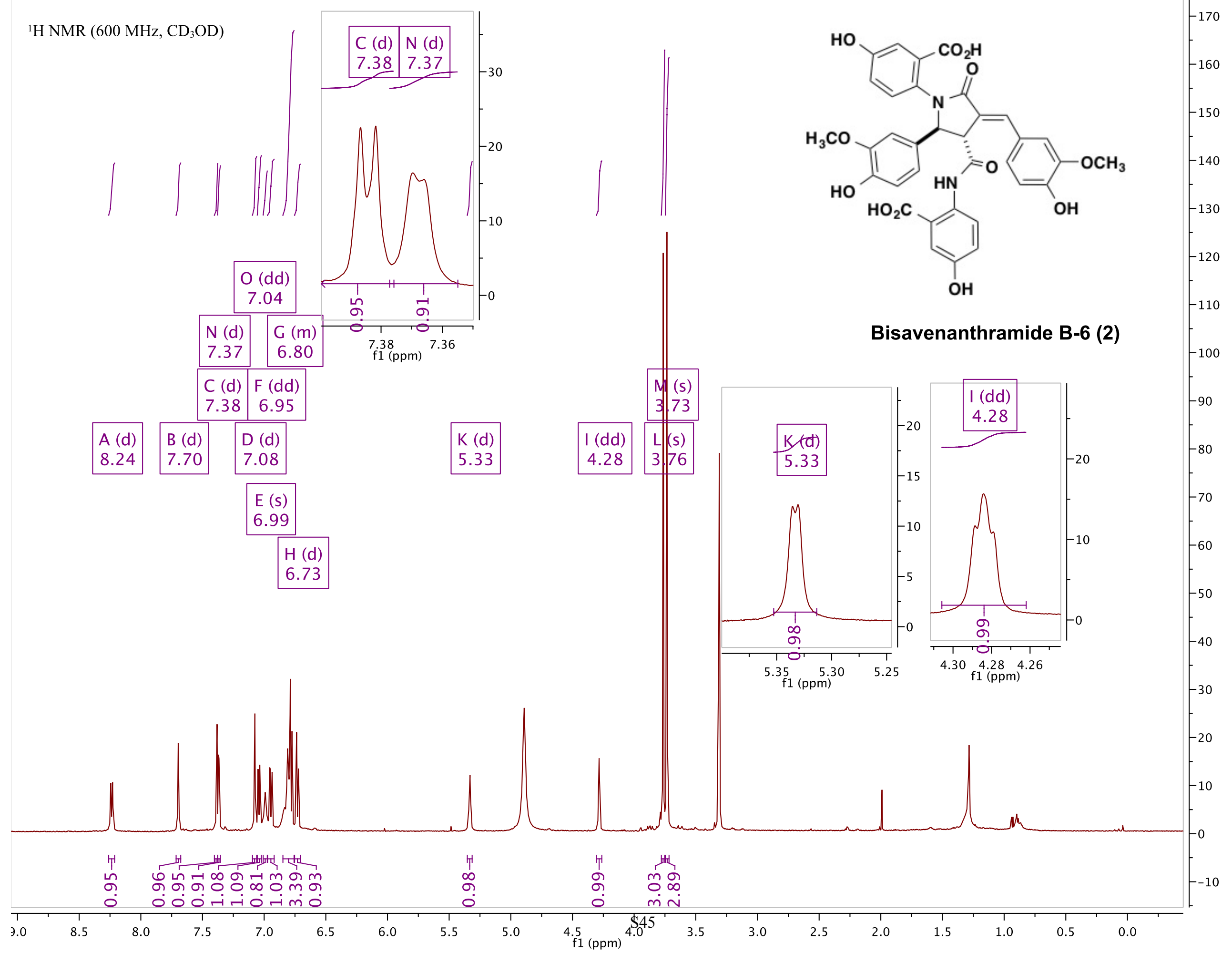




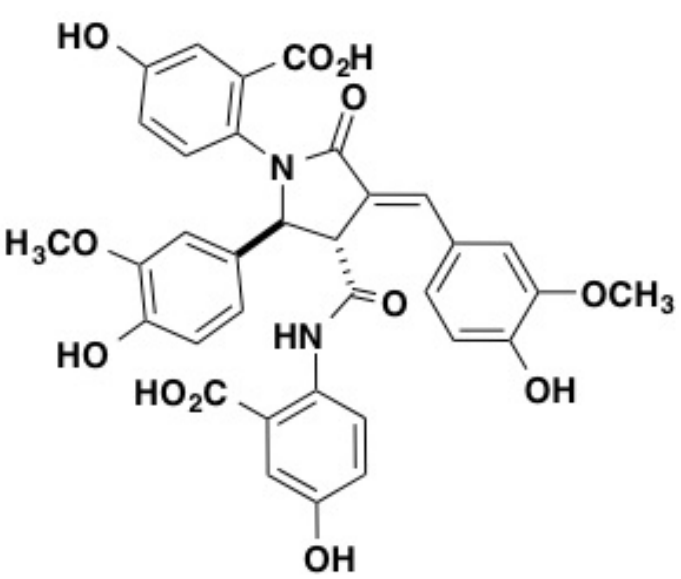

Bisavenanthramide B-6 (2)
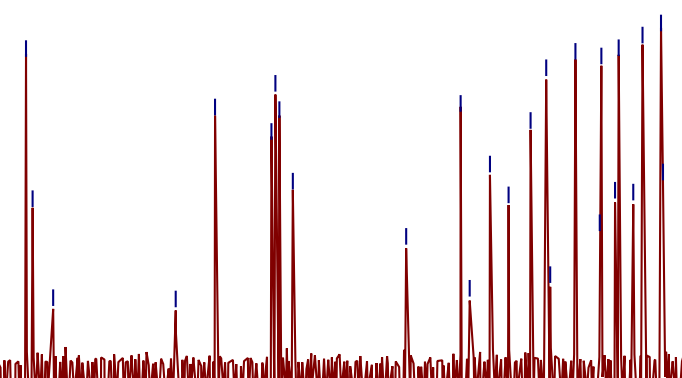